Elsevier required licence: (c) <2019>.

This manuscript version is made available under the CC-BY-NC-ND 4.0 license

http://creativecommons.org/licenses/by-nc-nd/4.0/

The definitive publisher version is available online at

https://www.journals.elsevier.com/journal-of-fluids-and-structures 


\title{
Vibroacoustic Responses of a Heavy Fluid Loaded \\ Cylindrical Shell Excited by a Turbulent Boundary Layer
}

\author{
Laurent Maxit ${ }^{1}$, Mahmoud Karimi ${ }^{2}$, Valentin Meyer $^{3}$, Nicole Kessissoglou ${ }^{4}$
}

1. Univ Lyon, INSA-Lyon, Laboratoire Vibrations-Acoustique (LVA), 25 bis, av. Jean Capelle, F-69621, Villeurbanne Cedex, France

e-mail: laurent.maxit@insa-lyon.fr

2. Centre for Audio, Acoustics and Vibration, University of Technology Sydney, Sydney, Australia e-mail: mahmoud.karimi@uts.edu.au

3. Naval Group Research, 199 av. Pierre-Gilles de Gennes, 83190 Ollioules, France e-mail: valentin.meyer@naval-group.com

4. School of Mechanical and Manufacturing Engineering, The University of New South Wales, Sydney, NSW 2052, Australia

e-mail: n.kessissoglou@unsw.edu.au

\begin{abstract}
A fully coupled structural-acoustic model of a cylindrical shell under external turbulent boundary layer excitation is herein developed. The numerical process requires computation of the wall pressure cross spectral density function as well as sensitivity functions for the fluid-loaded cylindrical shell. A semi-empirical model from literature is used to describe the wall pressure field induced by the turbulent boundary layer in the wavenumber-frequency domain. An analytical expression of the wall pressure field for a flat surface is adapted to describe the wall pressure field for a cylindrical surface. Circumferential sensitivity functions are derived using a wavenumber-point reciprocity principle. Results for the near-field and far-field acoustic pressure spectra are presented. Contributions of individual circumferential modes to the acoustic pressure spectra are examined, showing distinct trends below and above the ring frequency. The proposed
\end{abstract}


method is computationally efficient and provides an effective approach to investigate vibroacoustic responses for maritime platforms.

\section{Introduction}

Modelling the vibroacoustic behaviour of structures excited by a random pressure field such as a turbulent boundary layer (TBL) has significant benefit for naval applications. For example, a submerged marine vessel such as an autonomous underwater vehicle or a submarine is excited by pressure fluctuations due to turbulent flow induced by the vessel's movement through the water. Whilst interior and exterior noise from onboard machinery at low speeds and propeller noise at high speeds are dominant noise sources of an underwater vehicle, noise induced by a TBL is important in order to estimate the vessel self-noise. The self-noise radiated by a marine vessel can reduce the signal-to-noise ratio, leading to a reduction in performance of a passive sonar hull-mounted array. As such, understanding the vibroacoustic responses of a submerged vessel under TBL excitation can greatly assist in implementing mitigation strategies to minimize its radiated noise. The motivation of the current work is to develop a computationally efficient model of a fluid-loaded cylindrical shell under TBL excitation and to explore the physical mechanisms contributing to the near and far field structure-borne sound.

Theoretical, numerical and experimental studies have been extensively carried out to predict the vibroacoustic behaviour of plates in air excited by a turbulent flow field (for example, see Ciappi et al., 2014, 2018 and references therein). Strawderman (1969) semi-analytically modelled both an infinite plate and a finite plate under turbulence excitation. The infinite plate model was found to provide a good estimation of the plate vibration power spectra whilst the cross spectral properties of the vibration response were more accurately predicted by the finite plate model. The effect of heavy fluid loading on the two plate models excited by turbulent flow was then examined (Strawderman and Christman, 1971). The propagation speed in the infinite plate as well as resonances of the 
finite plate were shown to decrease compared with an equivalent plate in air. Davis (1971) compared the radiated sound power in air from a thin flexible panel excited by TBL wall pressure fluctuations using deterministic and statistical methods. In the deterministic method, the radiated sound power was estimated by directly summing over resonant modes from a modal analysis. The radiated power was also predicted using Statistical Energy Analysis assuming equipartition of energy between plate modes. At frequencies above the hydrodynamic coincidence frequency (which occurs when the plate flexural wavenumber is equal to the convective wavenumber), results from the two methods were shown to converge. Maury et al. (2002a, 2002b) proposed an analytical framework to predict the vibroacoustic responses of a panel excited by either a diffuse acoustic field or a fully developed turbulent flow. The structural displacement was represented by a Green's function representation in the wavenumber domain. An increase in flow velocity was observed to more rapidly increase the radiated sound pressure than the turbulent pressure. Hambric et al. (2004) used the finite element method (FEM) to examine the effect of different edge boundary conditions on the response of a flat plate under TBL excitation. They also proposed an approximate TBL model representing only the surface interaction, which was shown to work well for plates with clamped boundary conditions and at low wavenumber. De Rosa et al. (2008) analytically and numerically studied the structural response of a plate excited by a TBL using the modal expansion method and FEM. A scaling procedure was applied to both methods, leading to a significant reduction in simulation run time. Errico et al. (2019) proposed a numerical approach to estimate the sound transmission loss of complex flat, curved and cylindrical periodic structures, under acoustic or aerodynamic loads. The structural domain was modelled using the wave finite element method. The fluid-structure interaction was simulated in analogy to the acoustic wave excitation, discriminating among the different forcing models, using weighted wavenumber integration. For planar structures, the finite-size effects were taken into 
account using either the baffled window equivalence or asymptotic formulations. The approach was validated against experimental results. Ciappi et al. (2009) numerically and experimentally studied the response of a fluid-loaded plate under TBL excitation. The Corcos and Chase models were employed to predict the plate response. The Chase model was shown to provide good agreement between numerical and experimental results for the structure-borne acoustic responses. An approach based on sensitivity functions and reciprocity principles was employed by Marchetto et al. $(2017,2018)$ to model panels under diffuse acoustic field and TBL excitation. The vibroacoustic responses at any location either on the panel or in the acoustic medium was shown to depend on two quantities in the wavenumber domain; namely, the wall-pressure cross spectral density function of the excitation and the sensitivity function at that location. Mazzoni (2003) presented an analytical model based on wavenumber integration to approximate the vibroacoustic response of an elastic water-loaded plate excited by a TBL at low Mach number. Greater sensitivity of the plate modes in the subconvective region of the power spectrum of the turbulent excitation was observed.

The vibroacoustic responses of curved and composite panels under TBL excitation have also received attention. A deterministic approach combining modal expansion and receptance methods was developed by Liu (2008) to predict the radiated sound from aircraft panels subject to TBL excitation. The response of aerospace composite plates under turbulence induced vibration was numerically and experimentally investigated by Ciappi et al. (2016). They showed that at high Mach number, the aeroelastic effect on the panel response cannot be neglected, especially when composite materials are considered.

One of the earliest works on TBL excitation of cylindrical shells was presented by Norton and Bull (1984), to experimentally investigate the vibroacoustic responses of a thin cylindrical pipe excited by turbulent internal flow. At low frequencies where only acoustic plane waves can propagate in the pipe, peristaltic motion and resonant modes of 
the pipe wall were shown to contribute to the structural response. At higher frequencies, acoustic coincidence effects led to strong excitation of supersonic pipe modes, resulting in increasing pipe wall vibration and external radiation. Durant et al. (2000) numerically computed the structural and acoustic responses of a thin cylindrical pipe excited by turbulent internal flow using a boundary integral formulation. The wall pressure excitation was described by a Corcos model with the input parameters obtained from measurement of the cross spectral density of the wall pressure fluctuations. Recently, Li et al. (2017) studied random vibration analysis of an axially compressed cylindrical shell in air excited by an external TBL. The governing differential equations of the axially compressed cylindrical shell were derived and the eigenproblem was formed using the separation of variables technique. The cross spectrum density of the TBL was expanded as a Fourier series. Results for the shell responses were compared with those obtained using the modal decomposition method, showing good agreement.

Various studies have also been conducted to assess the acoustic performance of a double-walled cylindrical shell excited by external TBL pressure fluctuations. Tang et al. (1996) developed an analytical model for sound transmission in concentric cylindrical sandwich shells under TBL excitation of the outer shell surface. It was shown that the interior pressure was strongly affected by resonant modes above the hydrodynamic and acoustic coincidence frequencies at which noise can be transmitted efficiently. Zhou et al. (2015) analytically studied the effect of poroelastic material in the shell core on sound transmission in concentric cylindrical shells under external TBL excitation. The shell displacements and acoustic pressure in the interior, exterior and annular fluid domains were expressed by the modal expansion method. They showed that the shell responses due to TBL excitation formulated by the Corcos and Efimtsov models were similar at higher frequencies. In a similar study, Zhang et al. (2018) investigated the effect of microperforation at the inner wall on sound transmission in a double walled cylindrical 
shell under external TBL excitation.

In this paper, a numerical approach is presented to predict the vibroacoustic responses of a cylindrical shell immersed in a heavy fluid and excited by a TBL. The approach is computationally efficient, allowing the influence of individual circumferential modes on the structural and acoustic responses of the shell to be examined in detail. In the first step, the wall pressure field induced by the TBL in the wavenumber-frequency domain is described using a semi-empirical TBL model from literature. In the wavenumber domain, circumferential sensitivity functions which correspond to the structure-borne sound pressure from the cylindrical shell under unit wall plane waves are derived using a reciprocity relation between the acoustic pressure at a spatial location in the fluid and a radial force applied to the shell. The auto spectral density of the pressure in the acoustic near field and far field is then obtained. For insight into the physical mechanisms contributing to the near-field and far-field acoustic spectra, results are presented in terms of individual contributions of circumferential modes, in both the low and high frequency ranges below and above the ring frequency, and for increasing radial distance from the shell. The proposed method provides an effective tool to investigate noise and vibration of underwater vehicles.

\section{Methodology}

\subsection{Cylindrical shell under TBL excitation}

Let us consider an infinitely long thin cylindrical shell immersed in a fluid as shown in Fig. 1. A fully developed, stationary and homogeneous TBL excites the shell. It is assumed that the boundary layer is weakly coupled with the shell vibration and propagation of acoustic waves in the fluid is not affected by the flow. 

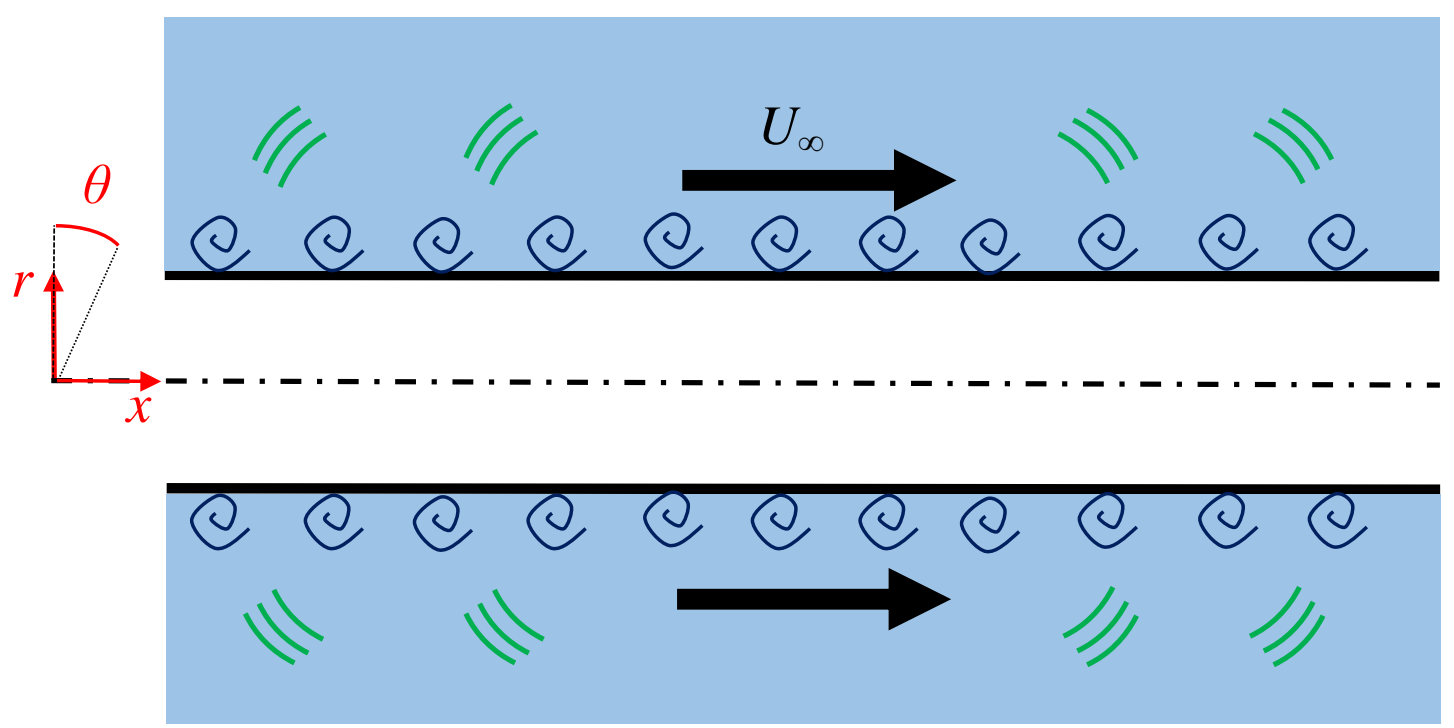

Figure 1. Schematic diagram of an infinitely long cylindrical shell immersed in heavy fluid and excited by a turbulent boundary layer.

The auto spectral density (ASD) of the radiated pressure from the cylindrical shell excited by the TBL is given by (Maury et al. 2002a)

$S_{p p}(x, \theta, r, \omega)=\int_{-\infty}^{\infty} \int_{-\infty}^{\infty} \int_{0}^{2 \pi} \int_{0}^{2 \pi}\left|H_{p / F}\left(x, \theta, r, x_{1}, \theta_{1}, \omega\right)\right|^{2} S_{p p}^{\mathrm{TBL}}\left(x_{2}-x_{1}, \theta_{2}-\theta_{1}, \omega\right) R^{2} d \theta_{1} d \theta_{2} d x_{1} d x_{2}$

where $H_{p / F}\left(x, \theta, r, x_{1}, \theta_{1}, \omega\right)$ is the frequency response function (FRF) of the radiated pressure at point $M$ in the fluid with coordinate $(x, \theta, r)$ for radial point force excitation of the shell at $M_{1} . S_{p p}^{\mathrm{TBL}}\left(x_{2}-x_{1}, \theta_{2}-\theta_{1}, \omega\right)$ is the cross spectral density (CSD) function of the wall pressure field between two points on the shell surface and is formulated in the following section.

\subsection{Wall pressure field}

The wall pressure field (WPF) induced by the TBL can be described by one of several semi-empirical TBL models proposed in the literature (for example, see Corcos 1963, Chase 1987, Goody 2004). Whilst these models have been established for planar structures such as a flat plate, they are assumed to accurately describe the WPF at the surface of a 
shell for low curvature (Lueptow 1988). For planar structures, the CSD function of the WPF in the wavenumber space, denoted by $\varphi_{p p}\left(k_{x}, k_{y}, \omega\right)$, is related to the CSD function of the WPF in the physical space, $S_{p p}^{\mathrm{TBL}}(x, y, \omega)$, as follows

$$
\begin{gathered}
\varphi_{p p}\left(k_{x}, k_{y}, \omega\right)=\int_{-\infty}^{\infty} \int_{-\infty}^{\infty} S_{p p}^{\mathrm{TBL}}(x, y, \omega) e^{-\mathrm{i} k_{x} x} e^{-\mathrm{i} k_{y} y} d x d y, \\
S_{p p}^{\mathrm{TBL}}(x, y, \omega)=\frac{1}{(2 \pi)^{2}} \int_{-\infty}^{\infty} \int_{-\infty}^{\infty} \varphi_{p p}\left(k_{x}, k_{y}, \omega\right) e^{\mathrm{i} k_{x} x} e^{\mathrm{i} k_{y} y} d k_{x} d k_{y},
\end{gathered}
$$

where $\omega$ is the angular frequency, and $k_{x}, k_{y}$ are wavenumber components in the $x$ - and $y$-directions, respectively. The $x$-axis represents the streamwise direction and the $y$-axis represents the spanwise direction.

For the cylindrical shell considered in this work, the spanwise direction $y$ corresponds to the shell circumferential direction $\theta$ and the WPF is circumferentially periodic. The wavenumber-frequency domain of the cylindrical shell is denoted by $\left(k_{x}, n\right)$, where $n$ is the circumferential mode number. The CSD function of the WPF in the wavenumber-frequency domain for a cylindrical shell, denoted by $\phi_{p p}\left(k_{x}, n, \omega\right)$ is related to $\varphi_{p p}\left(k_{x}, k_{y}, \omega\right)$ for a planar structure by

$$
\phi_{p p}\left(k_{x}, n, \omega\right)=\frac{1}{2 \pi R} \varphi_{p p}\left(k_{x}, k_{y}, \omega\right),
$$

where $R$ is the shell radius. The CSD function of the WPF for the cylindrical shell in the spatial domain is then obtained as

$$
S_{p p}^{\mathrm{TBL}}(x, \theta, \omega)=\frac{1}{2 \pi} \sum_{n=-\infty}^{+\infty}\left\{\int_{-\infty}^{+\infty} \phi_{p p}\left(k_{x}, n, \omega\right) e^{\mathrm{i} k_{x} x} d k_{x}\right\} e^{\mathrm{i} n \theta}
$$

\subsection{Circumferential sensitivity function}

Substituting Eq. (5) into Eq. (1), the ASD function of the radiated pressure can be rewritten in the following form: 


$$
S_{p p}(x, \theta, r, \omega)=2 \pi \sum_{n=-\infty}^{n=\infty} \int_{-\infty}^{\infty}\left|\tilde{H}_{p}\left(x, \theta, r, k_{x}, n, \omega\right)\right|^{2} \phi_{p p}\left(k_{x}, n, \omega\right) d k_{x},
$$

where

$$
\tilde{H}_{p}\left(x, \theta, r, k_{x}, n, \omega\right)=\frac{1}{2 \pi} \int_{-\infty}^{\infty} \int_{0}^{2 \pi} H_{p / F}\left(x, \theta, r, x_{1}, \theta_{1}, \omega\right) e^{-\mathrm{i} k_{x} x_{1}} e^{-\mathrm{i} n \theta_{1}} R d \theta_{1} d x_{1} .
$$

$\tilde{H}_{p}\left(x, \theta, r, k_{x}, n, \omega\right)$ corresponds to the radiated pressure at point $M$ when the shell is excited by a unit WPF given by $p(x, \theta)=e^{-\mathrm{i}\left(k_{x} x+n \theta\right)}$, and is termed the circumferential sensitivity function. The circumferential sensitivity function can be derived using a wavenumber-point reciprocity technique (Maxit and Denis 2013). The Lyamshev reciprocity principle as illustrated in Figure 2 states that the ratio of the radiated pressure $p$ at point $M$ to a radial point force $F$ located at point $M_{1}$ is equal to the ratio of the radial velocity $v$ at point $M_{1}$ to the volume velocity $Q_{v}$ at point $M$, that is,

$$
H_{p / F}\left(x, \theta, r, x_{1}, \theta_{1}, \omega\right)=H_{v / Q_{v}}\left(x_{1}, \theta_{1}, x, \theta, r, \omega\right) .
$$

Substituting Eq. (8) into Eq. (7) yields

$$
\tilde{H}_{p}\left(x, \theta, r, k_{x}, n, \omega\right)=\frac{1}{2 \pi} \int_{-\infty}^{\infty} \int_{0}^{2 \pi} H_{v / Q_{v}}\left(x_{1}, \theta_{1}, x, \theta, r, \omega\right) e^{-\mathrm{i} k_{x} x_{1}} e^{-\mathrm{i} n \theta_{1}} R d \theta_{1} d x_{1} .
$$

The circumferential sensitivity function can now be interpreted as the product of the radius $R$ with the Fourier transform of the shell radial velocity along the axial direction as well as a Fourier series decomposition in the circumferential direction, for the shell excited by an acoustic monopole located at point $M$ with coordinate $(x, \theta, r)$ and strength of unit volume velocity. Calculation of the circumferential sensitivity function requires the spectral radial velocity of the fluid-loaded cylindrical shell which is derived in the proceeding section. 


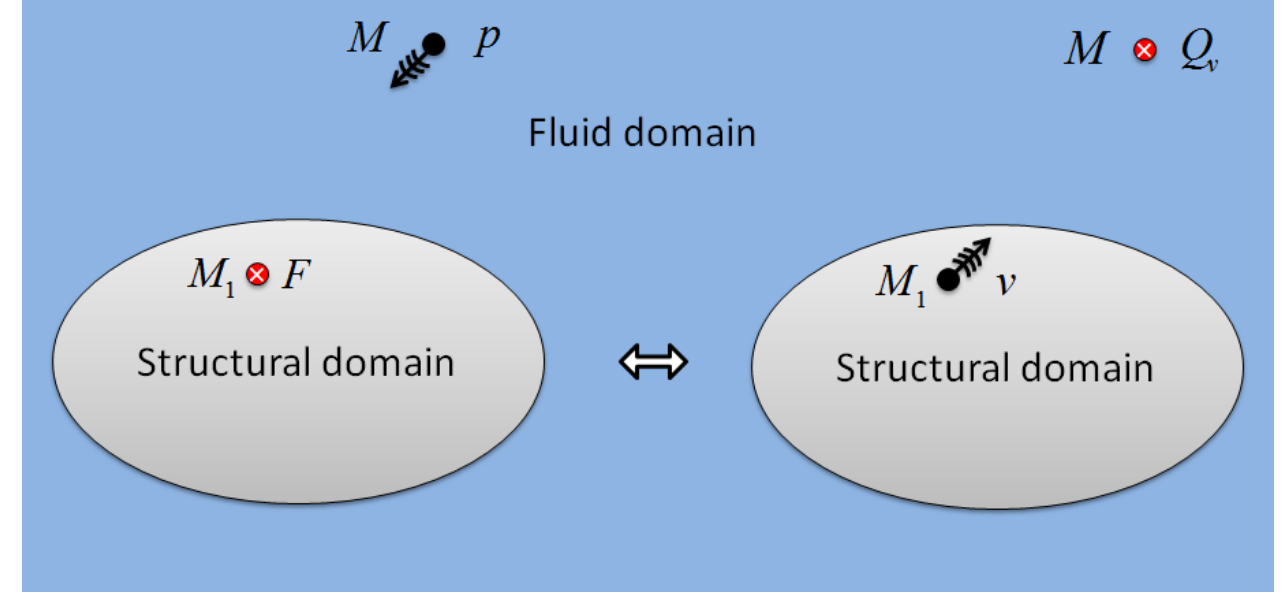

Figure 2. Illustration of the Lyamshev reciprocity principle between structural and fluid domains.

\subsection{Fluid-loaded cylindrical shell}

The cylindrical shell has radius $R$, thickness $h$, Young's modulus $E$, density $\rho_{s}$ and Poisson's ratio $v . U, V$, and $W$ are respectively the longitudinal, tangential and radial displacements of the shell. The shell is immersed in an infinite fluid domain of density $\rho_{f}$ and speed of sound $c_{f}$. The system is excited by a monopole source at point $M$ with coordinate $(x, \theta, r)$. The blocked wall pressure at $r=R$, corresponding to the induced WPF by the monopole when the shell is considered as rigid, is denoted by $p_{b}$. The structure-borne radiated wall pressure in the exterior acoustic domain is denoted by $p_{r}$.

Assuming the following time harmonic dependency $e^{\mathrm{i} \omega t}$, the Flügge equations of motion of the cylindrical shell are given by (Leissa 1973, Karczub 2006)

$$
\mathcal{L}(x, \theta)\left[\begin{array}{c}
U(x, \theta) \\
V(x, \theta) \\
W(x, \theta)
\end{array}\right]=\gamma\left(\left[\begin{array}{c}
0 \\
0 \\
p_{b}(x, \theta)
\end{array}\right]+\left[\begin{array}{c}
0 \\
0 \\
-p_{r}(x, \theta)
\end{array}\right]\right),
$$

where $\gamma=\frac{\left(1-v^{2}\right) R^{2}}{E h}$ and the elements of the spectral Flügge operator $\mathcal{L}(x, \theta)$ are 
listed in Appendix A. Applying both a Fourier series decomposition along the circumferential direction and a Fourier transform along the axial direction to Eq. (10) yields the shell equations of motion in the wavenumber domain as follows

$$
\tilde{\mathcal{L}}\left(k_{x}, n\right)\left[\begin{array}{c}
\tilde{U}\left(k_{x}, n\right) \\
\tilde{V}\left(k_{x}, n\right) \\
\tilde{W}\left(k_{x}, n\right)
\end{array}\right]=\gamma\left(\left[\begin{array}{c}
0 \\
0 \\
\tilde{p}_{b}\left(k_{x}, n\right)
\end{array}\right]+\left[\begin{array}{c}
0 \\
0 \\
-\tilde{p}_{r}\left(k_{x}, n\right)
\end{array}\right]\right) .
$$

The elements of spectral Flügge matrix $\tilde{\mathcal{L}}\left(k_{x}, n\right)$ in the wavenumber domain are listed in Appendix B.

Considering a monopole of unit volume velocity at point $M$ in the acoustic domain, the spectral blocked pressure at the external surface of the cylinder can be derived in a similar manner described by James (1982) as follows:

$$
\tilde{p}_{b}\left(k_{x}, n\right)=\frac{\mathrm{i} \omega \rho_{f}}{2 \pi k_{r} R} \frac{H_{n}^{(2)}\left(k_{r} r\right)}{H_{n}^{(2)^{\prime}}\left(k_{r} R\right)} e^{-\mathrm{i}\left(n \theta+k_{x} x\right)}
$$

where $H_{n}^{(2)}$ is the Hankel function of the second kind of order $n$, ()$^{\prime}$ denotes the derivative with respect to the argument, and $k_{r}$ is given by

$$
k_{r}=\left\{\begin{array}{l}
\sqrt{k_{f}^{2}-k_{x}^{2}} \text { if }\left|k_{x}\right| \leq k_{f}, \\
-\mathrm{i} \sqrt{k_{x}^{2}-k_{f}^{2}} \text { otherwise. }
\end{array}\right.
$$

In the acoustic domain, the radiated pressure from the vibrating shell satisfies the homogeneous Helmholtz equation (Junger and Feit 1986)

$$
\Delta p_{r}(x, \theta, r)+k_{f}^{2} p_{r}(x, \theta, r)=0,
$$

where $\Delta$ is the Laplacian operator in the cylindrical coordinate system. The kinematic condition at the cylindrical interface between the shell and fluid medium is given by (Junger and Feit 1986)

$$
\frac{\partial p_{r}}{\partial r}(x, \theta, R)=\rho_{f} \omega^{2} W(x, \theta) .
$$


Applying Fourier transforms to Eqs. (14) and (15), the spectral radiated wall pressure denoted by $\tilde{p}_{r}$ can be expressed in terms of the spectral radial displacement of the shell denoted by $\tilde{W}$ as well as the spectral fluid impedance, $\tilde{Z}_{f}$, as follows

$$
\begin{gathered}
\tilde{p}_{r}\left(k_{x}, n\right)=\tilde{Z}_{f}\left(k_{x}, n\right) \tilde{W}\left(k_{x}, n\right), \\
\tilde{Z}_{f}\left(k_{x}, n\right)=\frac{\rho_{f} \omega^{2}}{k_{r}} \frac{H_{n}^{(2)}\left(k_{r} R\right)}{H_{n}^{(2)^{\prime}}\left(k_{r} R\right)} .
\end{gathered}
$$

Substituting Eqs. (16) and (17) into Eq. (11) and inverting the matrix system yields the following expression for the spectral radial displacement:

$$
\tilde{W}\left(k_{x}, n\right)=\frac{\gamma \tilde{p}_{b}\left(k_{x}, n\right)\left(\tilde{Z}_{U U}\left(k_{x}, n\right) \tilde{Z}_{V V}\left(k_{x}, n\right)-\left(\tilde{Z}_{U V}\left(k_{x}, n\right)\right)^{2}\right)}{\Gamma\left(k_{x}, n\right)},
$$

where

$$
\begin{aligned}
& \Gamma\left(k_{x}, n\right)=\left|\tilde{\mathcal{L}}_{\mathrm{FL}}\left(k_{x}, n\right)\right|=\tilde{Z}_{U W}\left(\tilde{Z}_{U V} \tilde{Z}_{V W}-\tilde{Z}_{U W} \tilde{Z}_{V W}\right)+\tilde{Z}_{V W}\left(\tilde{Z}_{U W} \tilde{Z}_{U V}-\tilde{Z}_{V W} \tilde{Z}_{U U}\right)+ \\
& \left(\tilde{Z}_{W W}+\gamma \tilde{Z}_{f}\right)\left(\tilde{Z}_{U U} \tilde{Z}_{V W}-\tilde{Z}_{U V}^{2}\right)
\end{aligned}
$$

The spectral radial velocity of the fluid loaded cylindrical shell is $\mathrm{i} \omega \tilde{W}\left(k_{x}, n\right)$. Using Eqs. (12) and (18), the circumferential sensitivity function becomes

$\tilde{H}_{p}\left(x, \theta, r, k_{x}, n, \omega\right)=\frac{\mathrm{i} \omega \rho_{f} \gamma e^{-\mathrm{i}\left(n \theta+k_{x} x\right)}}{2 \pi k_{r}} \frac{H_{n}^{(2)}\left(k_{r} r_{s}\right)}{H_{n}^{(2)^{\prime}}\left(k_{r} R\right)} \frac{\left(\tilde{Z}_{U U}\left(k_{x}, n\right) \tilde{Z}_{V V}\left(k_{x}, n\right)-\left(\tilde{Z}_{U V}\left(k_{x}, n\right)\right)^{2}\right)}{\Gamma\left(k_{x}, n\right)}$

Substituting the circumferential sensitivity function given by Eq. (20) and the CSD function of the WPF given by Eq. (4) into Eq. (6) yields the ASD of the sound pressure from the cylindrical shell under TBL excitation.

\subsection{Wavenumber domain truncation}

The integral and summation of the ASD in Eq. (6) are with respect to infinite domains. In practice, these domains are truncated. The lowest frequency considered in this work is 
greater than the hydrodynamic coincidence frequency, which corresponds to the case when the flexural wavenumber of a plate denoted by $k_{p}$ with the same thickness and material properties of the cylindrical shell equals the convective wavenumber given by $k_{c}=\omega / U_{c}$, where $U_{c}$ is the convective velocity. The contributions of convective peaks to the acoustic pressure are then generally negligible (Hambric et al. 2004, Maxit 2016). As such, in the definition of the cut-off axial wavenumber, the convective wavenumber was not included. One can then define a cut-off axial wavenumber $k_{x, \mathrm{co}}$ from the characteristics of the shell and fluid as follows

$$
k_{x, \mathrm{co}}=\kappa_{x} \max \left(k_{p}, k_{f}\right) \text {, }
$$

where $k_{f}=\omega / c_{f}$ is the acoustic wavenumber and $\kappa_{x}$ is a margin coefficient (typically $\kappa_{x}=2$ ) (Maxit and Ginoux 2010). Similarly, the cut-off circumferential order $N_{\text {co }}$ can be defined by

$$
N_{\mathrm{co}}=\operatorname{int}\left[\kappa_{N} R \max \left(k_{p}, k_{f}\right)\right]+1
$$

where $\kappa_{N}$ is a second margin coefficient (typically $\kappa_{N}=1.5$ ) (Maxit and Ginoux 2010). Applying the cut-off axial wavenumber and the cut-off circumferential order, the ASD of the acoustic pressure given by Eq. (6) now becomes

$$
S_{p p}(x, \theta, r, \omega) \approx 2 \pi \sum_{n=-N_{\mathrm{co}}}^{n=N_{\mathrm{co}}} \int_{k_{x, \mathrm{co}}}^{k_{x, \mathrm{co}}}\left|\tilde{H}_{p}\left(x, \theta, r, k_{x}, n, \omega\right)\right|^{2} \phi_{p p}\left(k_{x}, n, \omega\right) d k_{x},
$$

where the integral may be estimated numerically using the rectangular rule. To study the individual contributions of the circumferential modes to the ASD, Eq. (23) can be written as $S_{p p}(x, \theta, r, \omega) \approx \sum_{n=0}^{N_{\mathrm{co}}} C_{n}$ with $C_{n}$ the contribution of the $n^{\text {th }}$ mode given by 


$$
C_{n}=2 \pi \varepsilon_{n} \int_{-k_{x, c o}}^{k_{x, c o}}\left|\tilde{H}_{p}\left(x, \theta, r, k_{x}, n, \omega\right)\right|^{2} \phi_{p p}\left(k_{x}, n, \omega\right) d k_{x},
$$

where $\varepsilon_{n}=\left\{\begin{array}{l}1 \text { for } n=0 \\ 2 \text { for } n \in\left[1, N_{\mathrm{co}}\right]\end{array}\right.$

\section{Numerical results}

An infinite cylindrical shell submerged in water with a radius of $R=5 \mathrm{~m}$ and thickness of $h=0.05 \mathrm{~m}$ is studied here. The shell is made of steel with density $\rho_{s}=7800 \mathrm{~kg} / \mathrm{m}^{3}$, Young's modulus $E=2.1 \times 10^{11} \mathrm{~Pa}$, Poisson's ratio $v=0.3$, and structural loss factor $\eta=0.02$. The density and speed of sound in the water are $\rho_{f}=1000 \mathrm{~kg} / \mathrm{m}^{3}$ and $c_{f}=1500 \mathrm{~m} / \mathrm{s}$, respectively. A freestream velocity of $U_{\infty}=6 \mathrm{~m} / \mathrm{s}$ was assumed, which forms a homogeneous fully developed turbulent boundary layer on the surface of the shell. A friction velocity of $v_{\tau}=1 \mathrm{~m} / \mathrm{s}$ and boundary layer thickness of $\delta=0.15 \mathrm{~m}$ was used in the numerical calculations. The Chase TBL model, valid for $\omega \delta / U_{\infty}>>1$, was employed to model the CSD function of the wall pressure field. The expression for the CSD of the Chase model can be found in Appendix C. Using the aforementioned TBL parameters, the Chase model can be confidently implemented for frequencies greater than $10 \mathrm{~Hz}$. As the original spectrum given by the Chase model is a two-sided angular frequency spectrum, it was multiplied by $4 \pi$ to convert it to a one-sided frequency spectrum. In addition, due to the difference between the Fourier conventions used in Chase (1987) and the current work, the wall pressure spectrum was multiplied by $(2 \pi)^{3}$ to have the same Fourier convention.

The vibroacoustic responses of the shell were calculated for a frequency range between $10 \mathrm{~Hz}$ to $1000 \mathrm{~Hz}$. Three distinct frequencies relevant to the current analysis correspond to the hydrodynamic coincidence frequency, the ring frequency and the acoustic coincidence frequency, corresponding to the frequency at which the equivalent plate flexural wavenumber is equal to the acoustic wavenumber. For the parameters chosen here, the 
hydrodynamic coincidence frequency is $0.036 \mathrm{~Hz}$ and the critical frequency is $4561 \mathrm{~Hz}$. As such, only the ring frequency of the cylindrical shell, occurring at $173 \mathrm{~Hz}$, lies in the frequency range of interest. At the ring frequency, the shell resonates as a ring due to the fact that longitudinal waves travel in the shell with a wavelength equal to the shell circumference. Below the ring frequency, the effect of the shell curvature is important and most of the shell vibrational energy is in stretching. Above the ring frequency, the shell vibrates in a similar way to a flat plate and most of the energy is in bending (Fahy and Gardonio, 2007).

\subsection{Spectral displacement of the fluid loaded shell}

The vibratory response of the fluid loaded shell in the wavenumber domain under point force excitation is initially examined. The radial point force $p_{F}(x, \theta)=\delta(x) \delta(\theta)$ is applied at $(x, \theta)=(0,0)$, thereby exciting all axial wavenumber components and circumferential modes with the same magnitude. The Fourier transform of the pressure distribution due to the radial point force is $\tilde{p}_{F}\left(k_{x}, n\right)=\frac{1}{2 \pi}$. The spectral displacement of the shell is then given by

$$
\tilde{W}_{F}\left(k_{x}, n\right)=\frac{\tilde{p}_{F}\left(k_{x}, n\right) \gamma\left(\tilde{Z}_{U U}\left(k_{x}, n\right) \tilde{Z}_{V V}\left(k_{x}, n\right)-\left(\tilde{Z}_{U V}\left(k_{x}, n\right)\right)^{2}\right)}{\Gamma\left(k_{x}, n\right)}
$$

which differs from Eq. (18) with the term $\tilde{p}_{F}$ instead of $\tilde{p}_{b}$. Figure 3 presents the spectral shell displacement at three discrete circumferential modes corresponding to $n=0$, $n=1$ and $n=7$. In each figure, the acoustic wavenumber $k_{f}$, denoted by a black dashed line, as well as the flexural wavenumber of an equivalent fluid loaded plate denoted by a white dashed line, are also shown. The flexural wavenumber of a fluid loaded plate was calculated using $k_{p, F L}=\left(k_{p}^{4}+\omega^{2} \rho_{f} /\left(D \sqrt{k_{p}^{2}-k_{f}^{2}}\right)\right)^{1 / 4}$, where $k_{p}=\left(\omega^{2} \rho_{s} h / D\right)^{1 / 4}$ is the 
flexural wavenumber of the in vacuo plate and the second term in brackets is the added mass to take into account the fluid loading effects. Figure 3(a) shows that at low frequencies, the axisymmetric $n=0$ mode is a subsonic wave (with axial wavenumber components greater than the acoustic wavenumber), whereby the axial wavenumber is exponentially close to the acoustic wavenumber. As frequency increases, the wavenumber increases and asymptotes to that of the plate flexural wavenumber above the ring frequency. This finding has been previously reported by Photiadis (1990). For the $n=1$ bending mode in Figure 3(b), the strong effect of the shell curvature at low frequencies can be observed. Similar to the $n=0$ mode, the axial wavenumber components of the $n=1$ mode are subsonic and above the ring frequency, asymptote to the plate flexural wavenumber. The cut-on frequencies of the quasi-compressional and shear waves can also be observed at around $100 \mathrm{~Hz}$ and $170 \mathrm{~Hz}$, respectively. The first circumferential mode to cut on in the frequency range from $10 \mathrm{~Hz}$ to $1 \mathrm{kHz}$ corresponds to the $n=7$ mode at a cut-on frequency of $14 \mathrm{~Hz}$ (Figure 3(c)). Here it is observed that the spectral displacement is close the plate flexural wavenumber. Similar findings for subsonic waves of a fluid-loaded cylindrical shell to those presented here have been previously observed in the dispersion graphs reported by Scott (1988). 


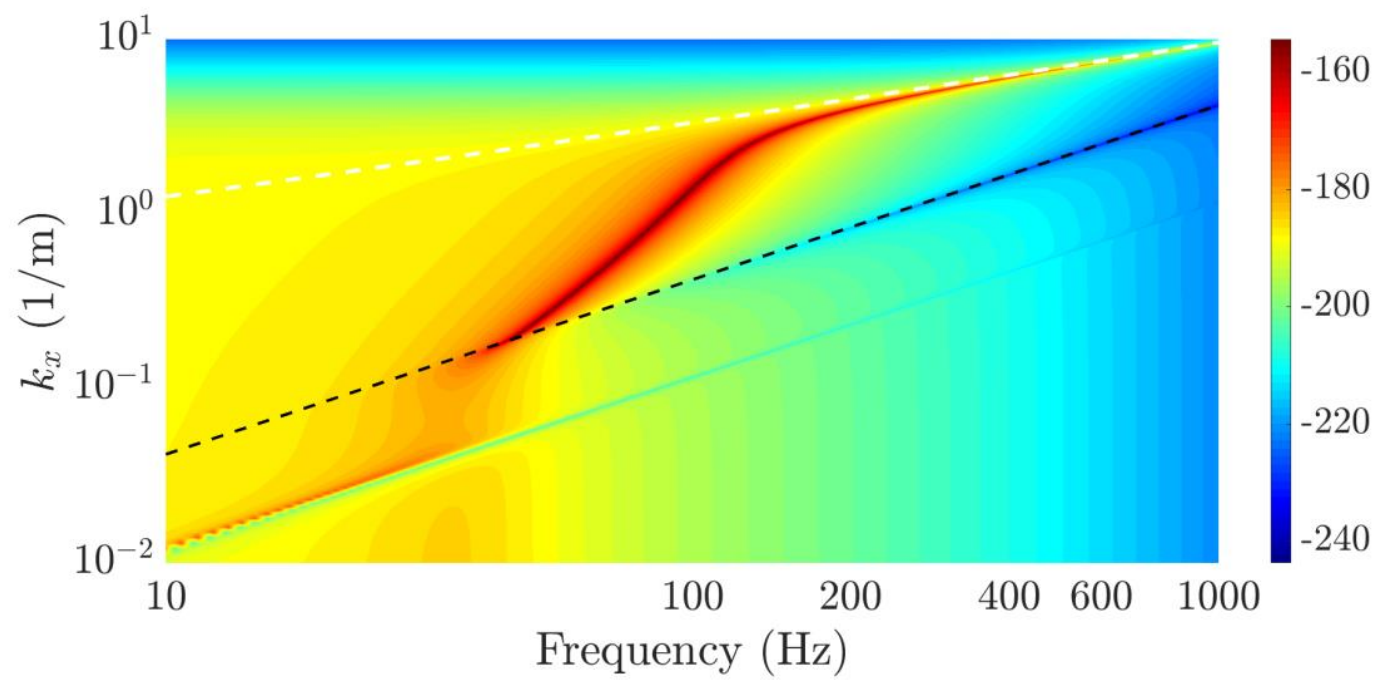

(a)

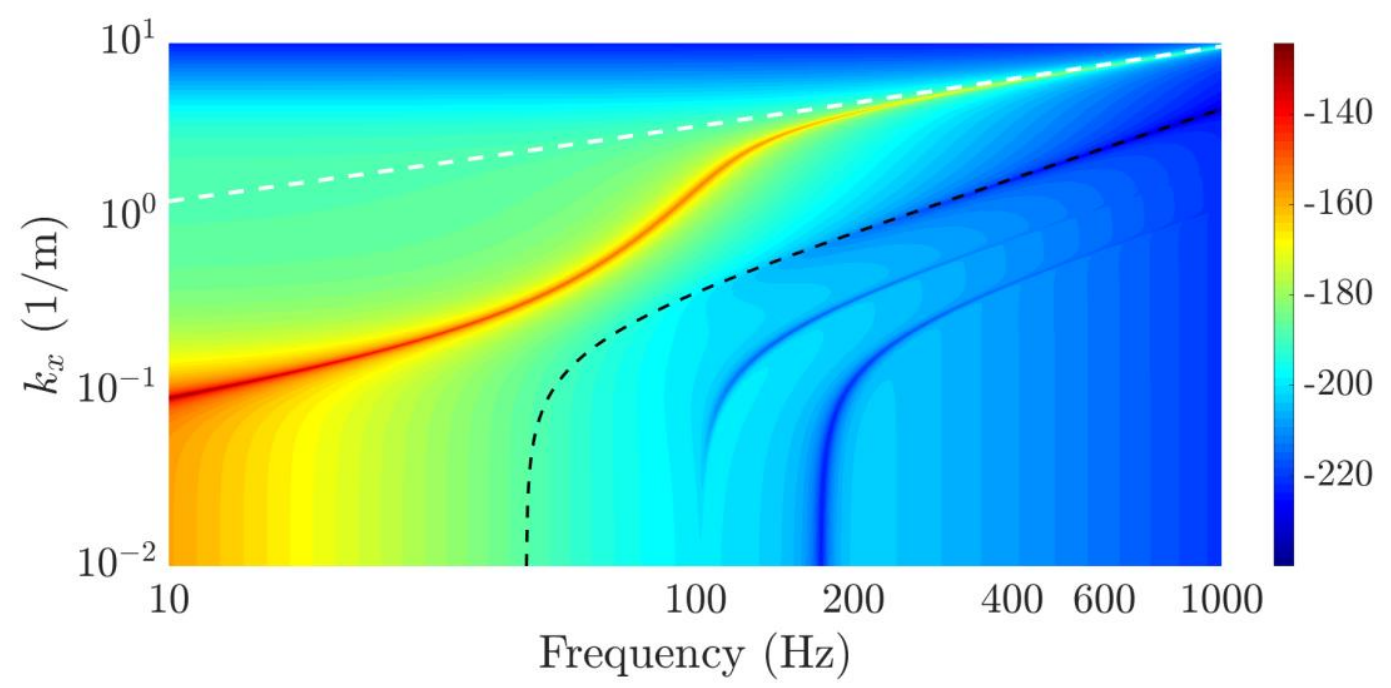

(b)

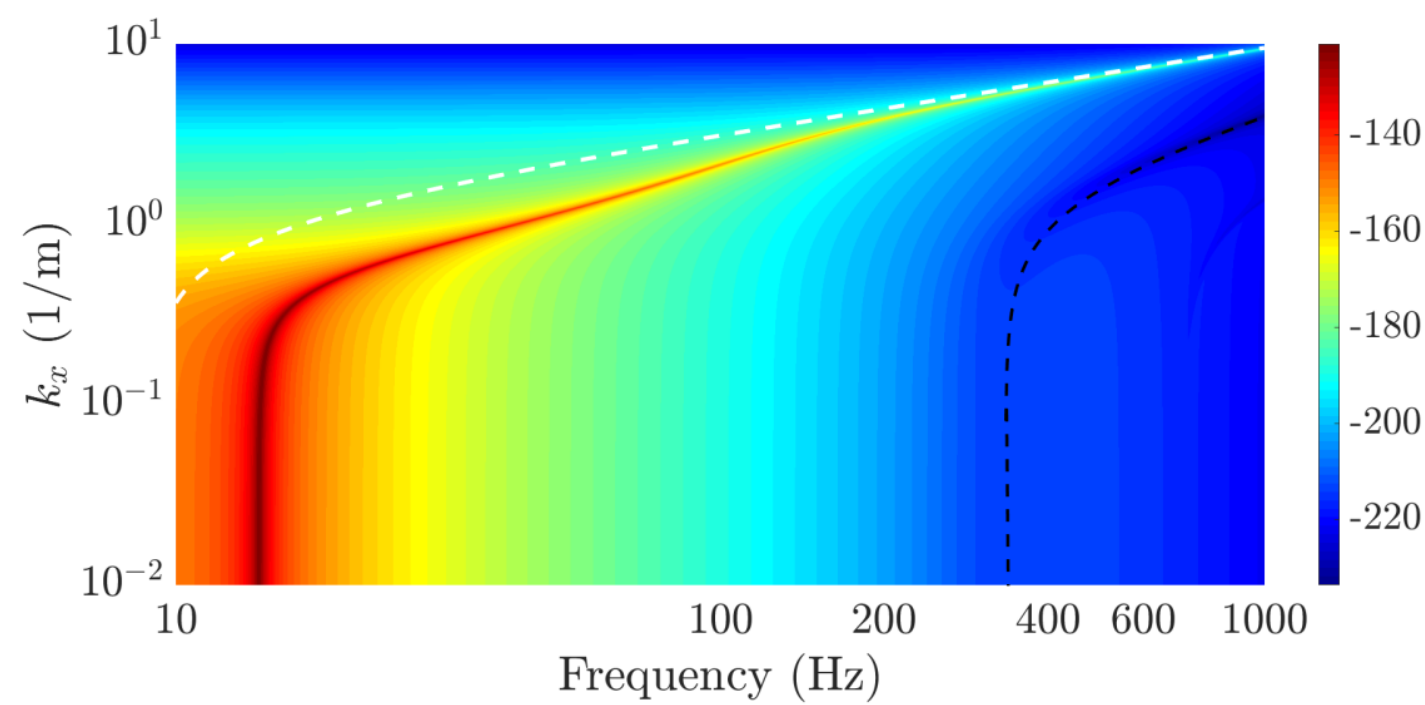

(c)

Figure 3. Spectral displacement of the shell under radial point force excitation for (a) $n=0$, (b) $n=1$ and (c) $n=7$. The black dashed line denotes the acoustic wavenumber and the white dashed line corresponds to the flexural wavenumber of an equivalent fluid loaded plate. 


\subsection{Near field acoustic spectra}

Figure 4 presents the ASD function of the acoustic pressure in the near field as a function of frequency. Contributions by individual circumferential modes to the acoustic spectrum are also shown. The $n=0$ and $n=1$ modes do not cut on at specific frequencies in the frequency range of interest, as shown previously in Figures 3(a) and 3(b), respectively. Below the ring frequency, the subsonic waves associated with these modes have negligible contribution to the near-field acoustic response. The distinct peaks in the acoustic spectra correspond to $n \geq 2$ shell circumferential modes that cut on at specific frequencies. The first peak in Figure 4 corresponds to the $n=7$ mode at a cut on frequency of $14 \mathrm{~Hz}$, as shown previously in Figure 3(c) (whereby the $n=2-6$ modes cut on below $10 \mathrm{~Hz}$ ). Below their respective cut-on frequency, the $n \geq 2$ modes have very little contribution to the near-field acoustic spectrum. In contrast, at respective cut on frequencies that occur below the ring frequency, these modes have a significant effect on the near-field acoustic response. Above the ring frequency, all modes have similar contribution to the total acoustic spectrum. The $n=0$ mode asymptotes to a lower value by a constant amount of around $3 \mathrm{~dB}$, associated with the fact that $C_{n}$ in Eq. (24) is defined as the sum of the contribution corresponding to $[-n, n]$ for all $n \geq 1$ modes and as such, is twice the value of $C_{0}$.

Circumferential sensitivity functions for the cylindrical shell in the near field $(z=0.1$ m) are presented in Figure 5 at three distinct frequencies, corresponding to $10 \mathrm{~Hz}, 100 \mathrm{~Hz}$ and $1000 \mathrm{~Hz}$. Two semi-ellipse curves are also depicted in Figure 5 whereby similar to Figure 3, the black dashed line is associated with the acoustic wavenumber represented by $\left\{k_{x}, R \sqrt{k_{f}^{2}-k_{x}^{2}}\right\}$ where $k_{x} \in\left[-k_{f}, k_{f}\right]$, and similarly, the white dashed line is associated with the flexural wavenumber of an equivalent fluid-loaded plate represented by $\left\{k_{x}, R \sqrt{{k_{p, F L}^{2}-k_{x}^{2}}^{2}}\right\}$ where $k_{x} \in\left[-k_{p, F L}, k_{p, F L}\right]$. Figure 5 shows that in the acoustic near 
field, the maxima of the sensitivity function are located on the semi-ellipse that corresponds to flexural vibration of an equivalent fluid loaded plate. These values are essentially the roots of the characteristic equation given by Eq. (19). Figure 5(a) shows that a maximum of 6 circumferential modes contribute to the acoustic spectrum at $10 \mathrm{~Hz}$ (the $n=7$ mode cuts on at $14 \mathrm{~Hz}$ as shown previously in Figure 4). At $100 \mathrm{~Hz}$ in Figure 5(b), it can be observed that around 15 circumferential modes contribute to the acoustic pressure whilst at $1000 \mathrm{~Hz}$ (Figure 5(c)), around 50 circumferential modes contribute to the pressure spectrum. The subsonic waves associated with the wavenumber region outside the acoustic semi-ellipse correspond to evanescent waves that propagate close to the cylinder and only contribute to the near field pressure spectrum. These results are consistent with the cut on modes discussed previously in Figure 4. Figure 5(c) also shows that as frequency increases, the contribution by $C_{n}$ for the lowest order circumferential modes increases attributed to the acoustic filtering effect, which will be discussed subsequently.

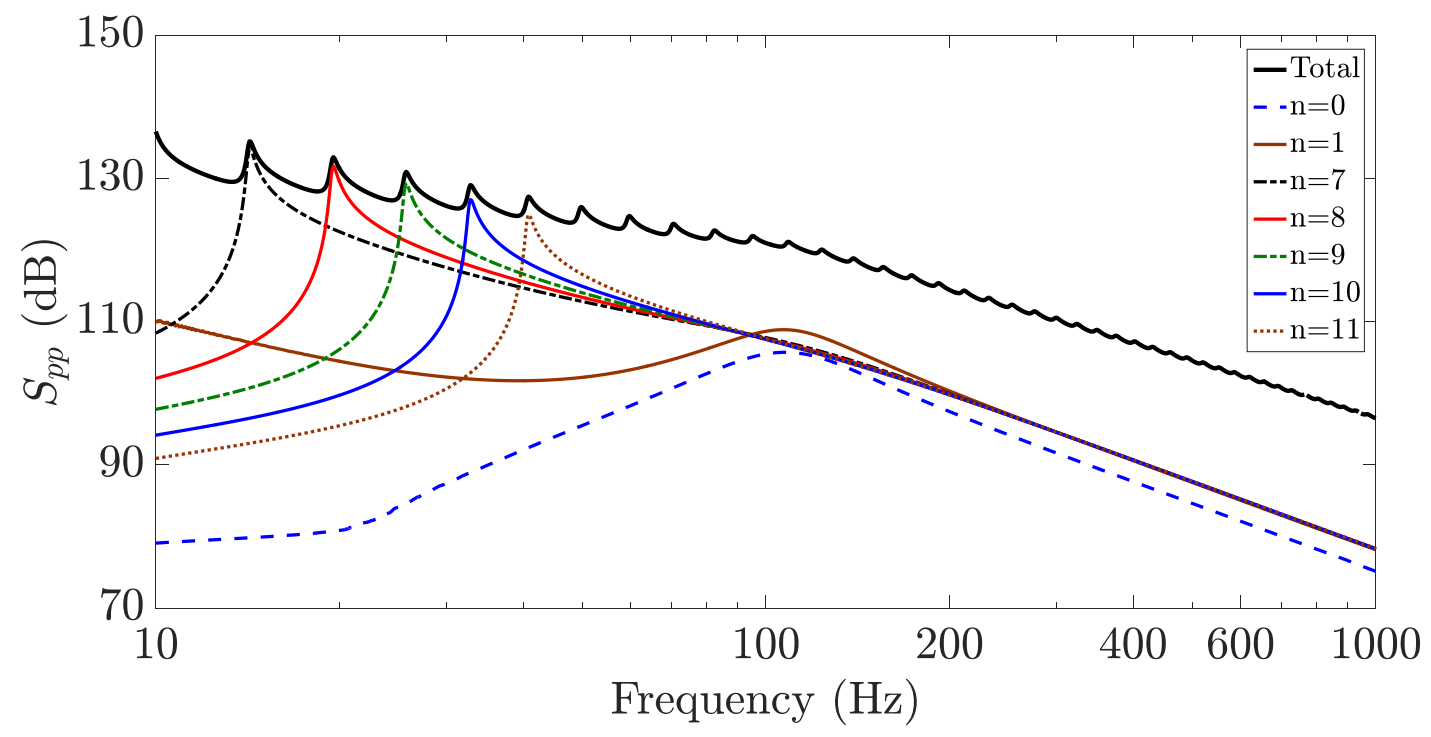

Figure 4. Auto spectral density of the acoustic pressure at $z=0.1 \mathrm{~m}(\mathrm{~dB}$ ref. $1 \mu \mathrm{Pa} / \sqrt{\mathrm{Hz}})$. 


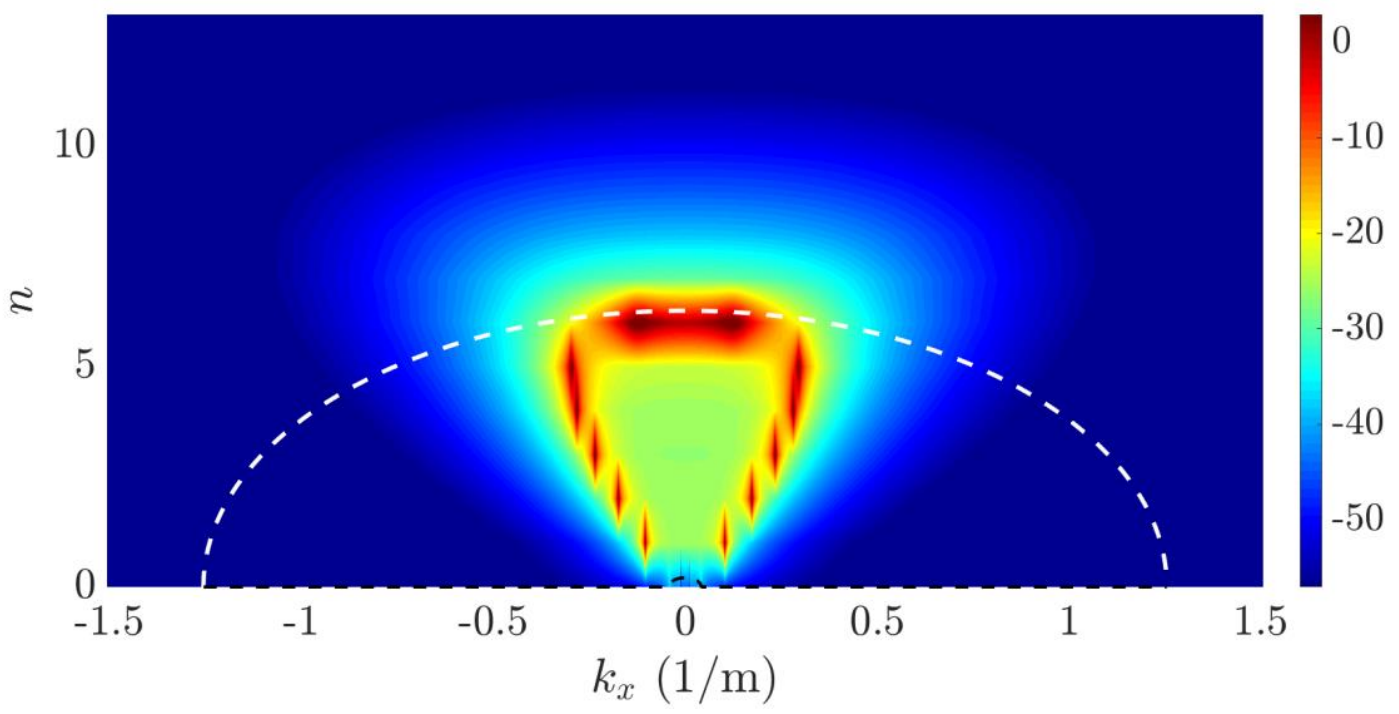

(a)

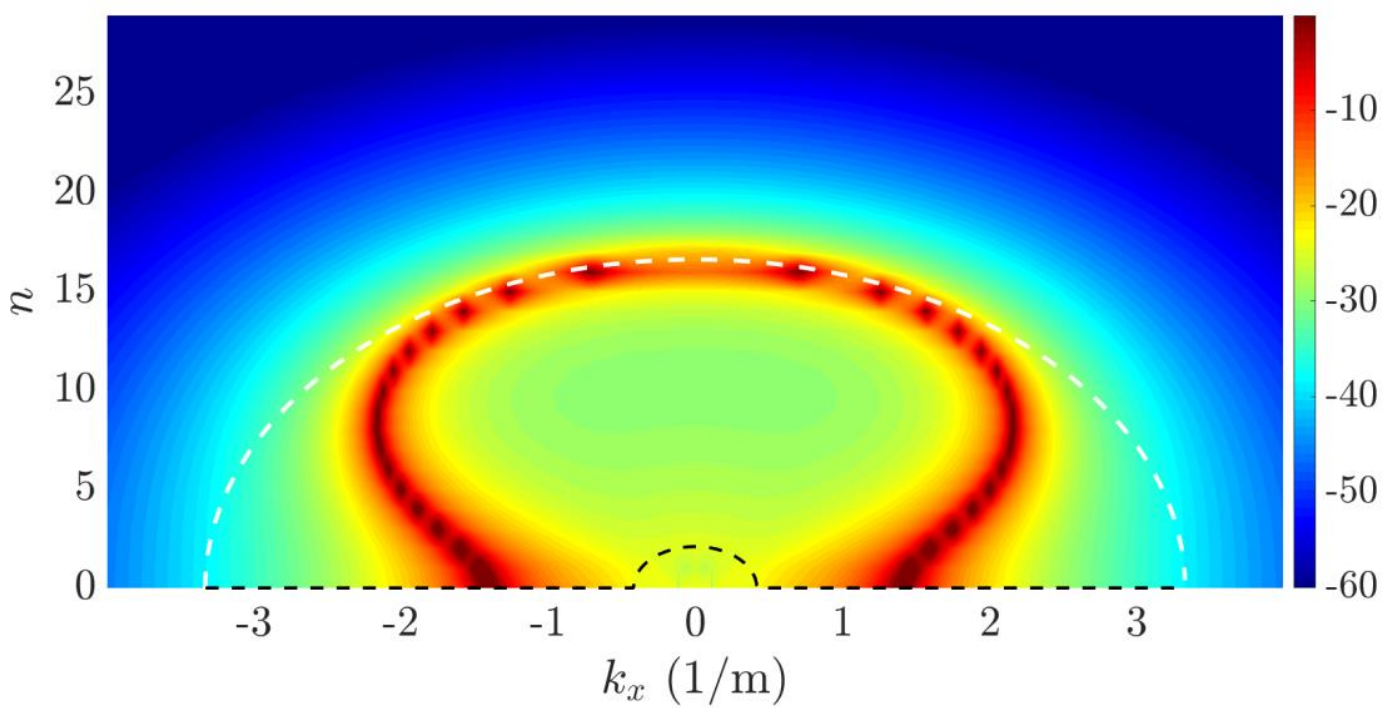

(b)

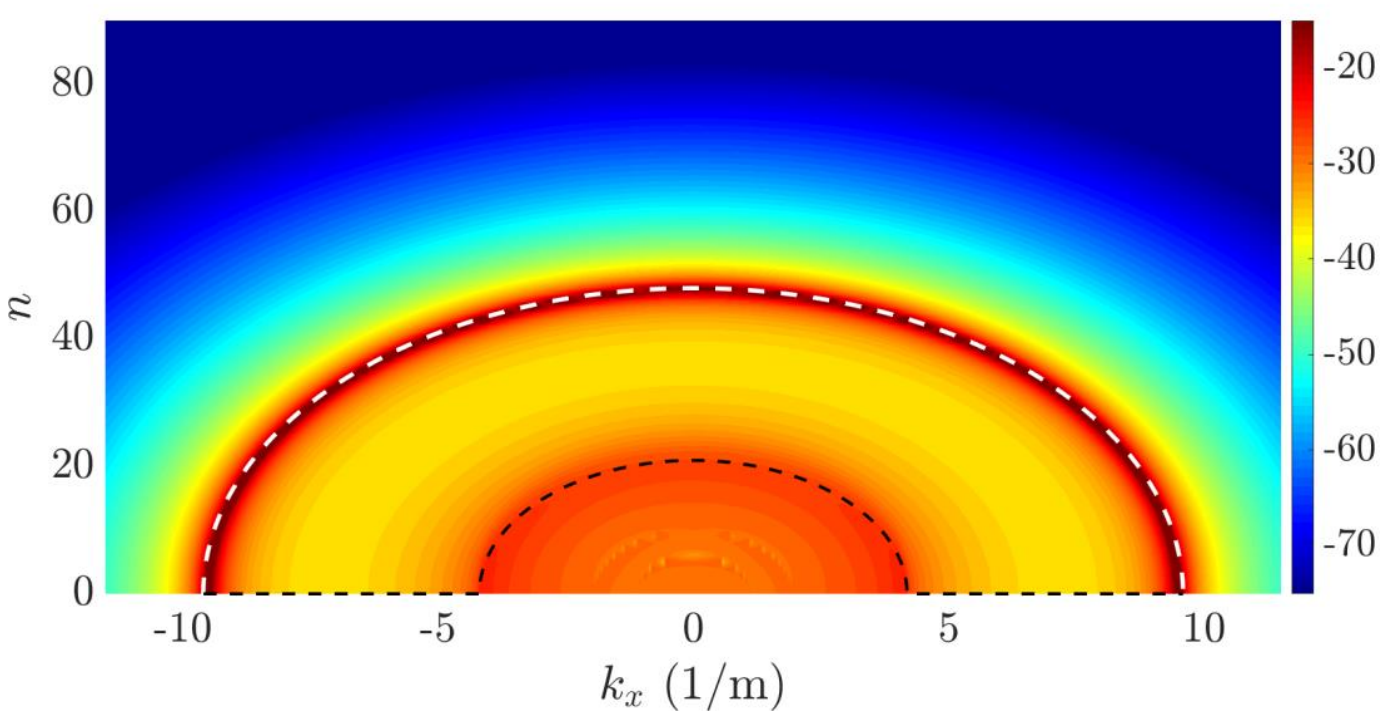

(c)

Fig 5. Circumferential sensitivity function in the acoustic near field $(\mathrm{z}=0.1 \mathrm{~m})$ at (a) $10 \mathrm{~Hz}$, (b) $100 \mathrm{~Hz}$ and (c) $1000 \mathrm{~Hz}$. 


\subsection{Far field acoustic spectra}

The ASD function of the acoustic pressure and the corresponding circumferential sensitivity function are herein examined at increasing radial distances from the shell. Figure 6(a)-(c) presents the acoustic pressure as a function of frequency and including contributions by individual circumferential modes, at radial distances of $z=10 \mathrm{~m}, z=100 \mathrm{~m}$ and $z=1000 \mathrm{~m}$, respectively. It can be observed that with increasing radial distance from the shell, the lowest order circumferential modes and in particular the $n=0$ mode, play an increasingly dominant role in the radiated pressure at low frequencies. This is attributed to the acoustic filtering effect associated with the blocked pressure $\tilde{p}_{b}$ given by Eq. (12), and can be more clearly observed in the sensitivity function in Figure 7 (see also Williams et al. 1990). At very low frequencies, Figure 7 shows that only the lowest order modes occur within the supersonic wavenumber region with increasing distance from the shell, whereby all other wavenumber components are completely attenuated by the acoustic medium. Figure 8 shows that at a higher frequency (above the ring frequency), the same number of modes contribute to the acoustic spectrum for all three radial distances.

Figure 9 presents the ASD function of the radiated pressure at different radial distances from the shell surface. When the observation point is located in the near field $(z=0.1 \mathrm{~m}$ and $z=1 \mathrm{~m}$ ), the pressure spectra exhibits a large number of fluctuations at low frequencies corresponding to the $n \geq 7$ modes for the frequency range considered here. When the receiver point is moved further away from the shell, the pressure spectrum becomes smoother and beyond a certain distance, remains almost constant with only slight variation within the frequency range of interest, associated with a similar number of modes contributing to the total spectrum as shown in Figure 8. In addition, the difference between spectrum levels becomes constant. An offset of approximately $10 \mathrm{~dB}$ between the spectrum levels at $z=10 \mathrm{~m}$, $z=100 \mathrm{~m}$ and $z=1000 \mathrm{~m}$ at higher frequencies can be observed, consistent with the $1 / r$ decay law associated with cylindrical spreading (noting $r=R+z$ ). 


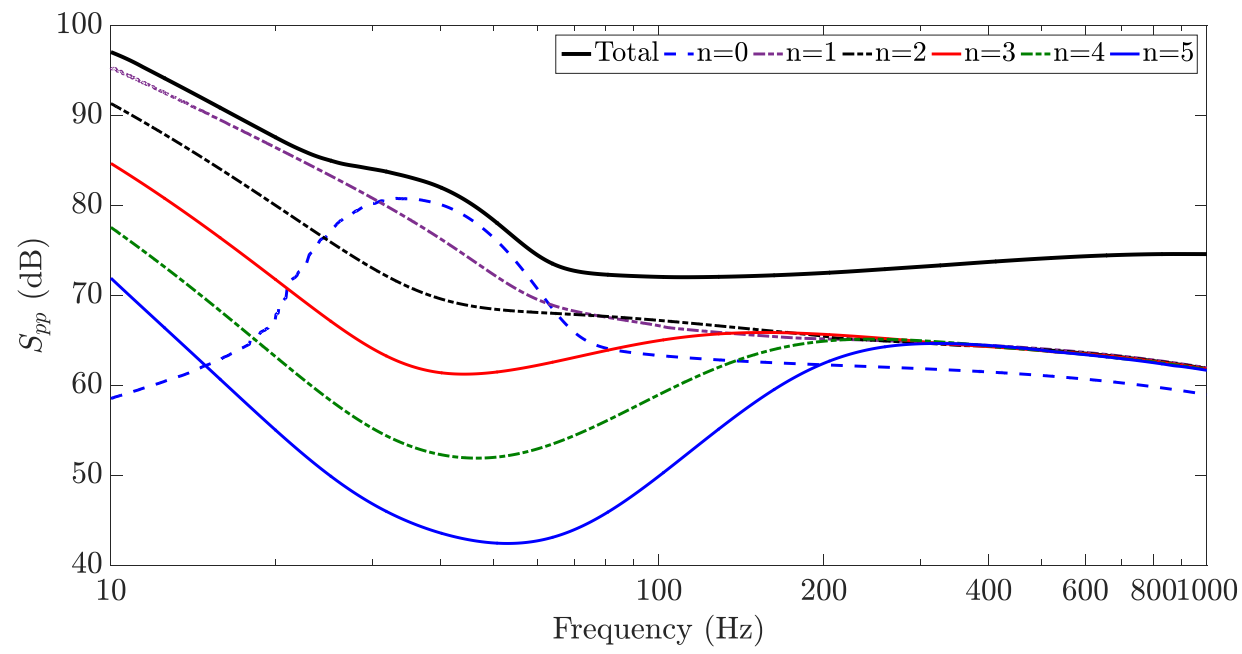

(a)

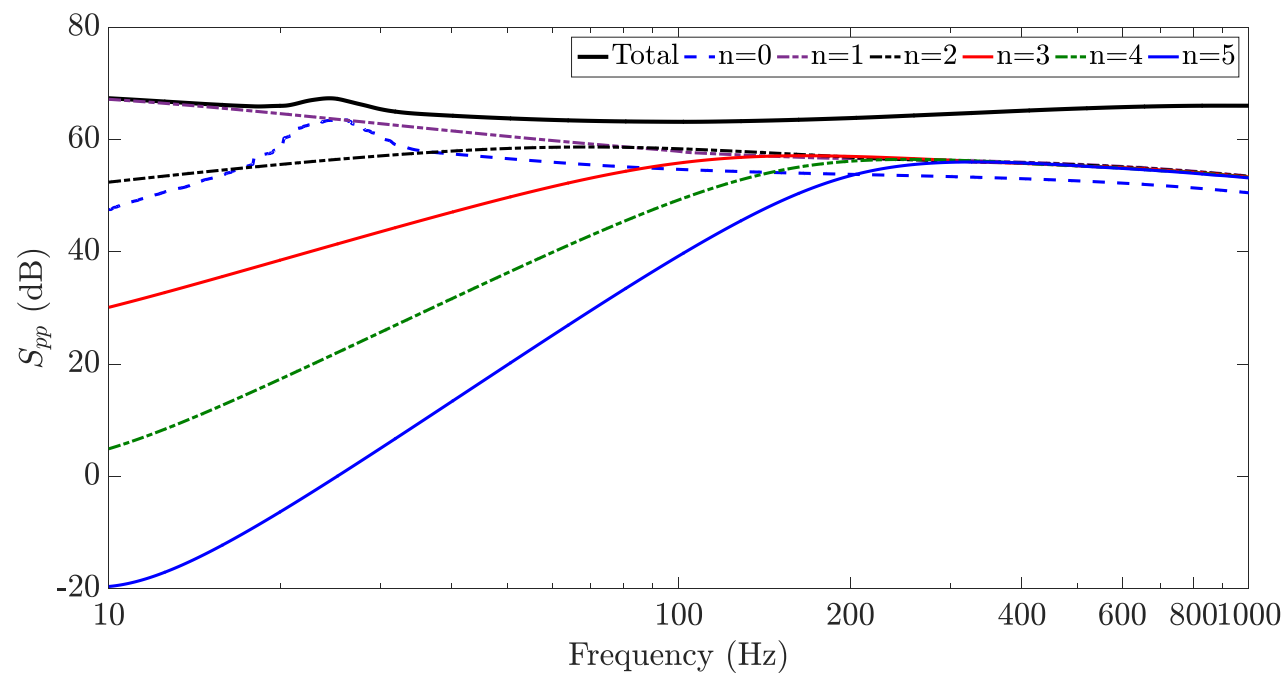

(b)

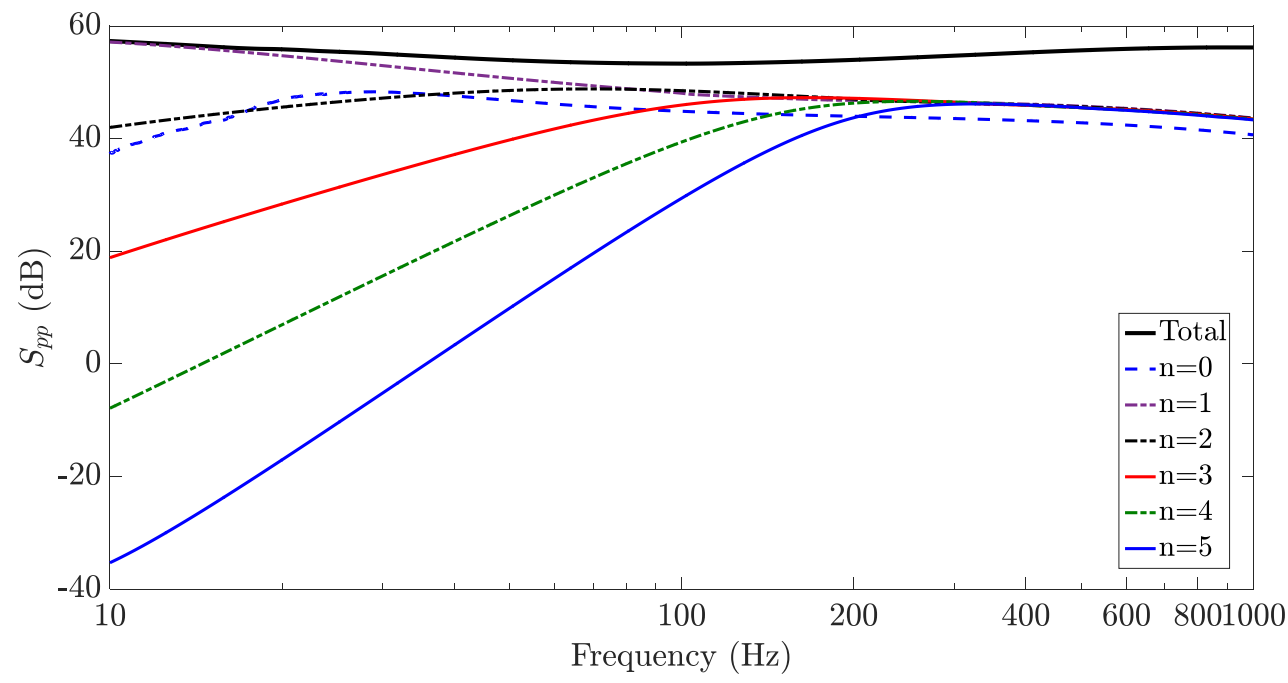

(c)

Figure 6. Auto spectral density of the acoustic pressure at (a) $z=10 \mathrm{~m}$, (b) $z=100 \mathrm{~m}$ and (c) $\mathrm{z}=1000 \mathrm{~m}(\mathrm{~dB}$ ref. $1 \mu \mathrm{Pa} / \sqrt{\mathrm{Hz}})$. 


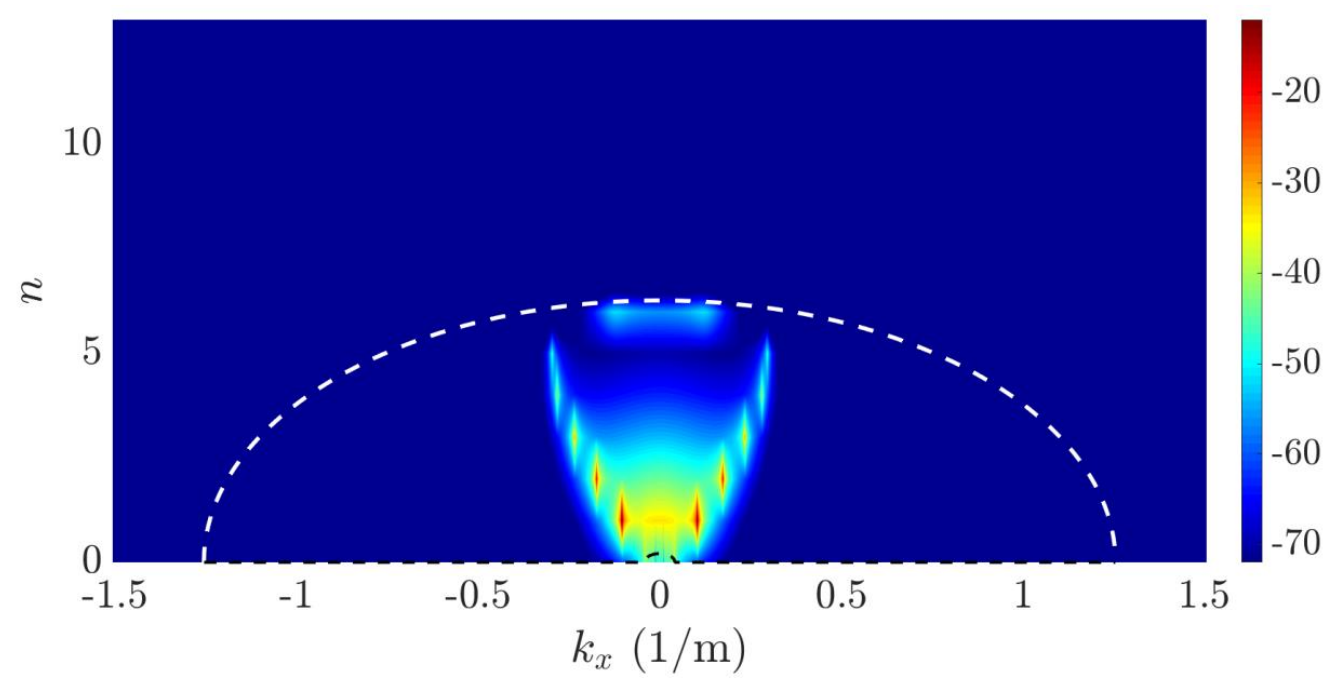

(a)

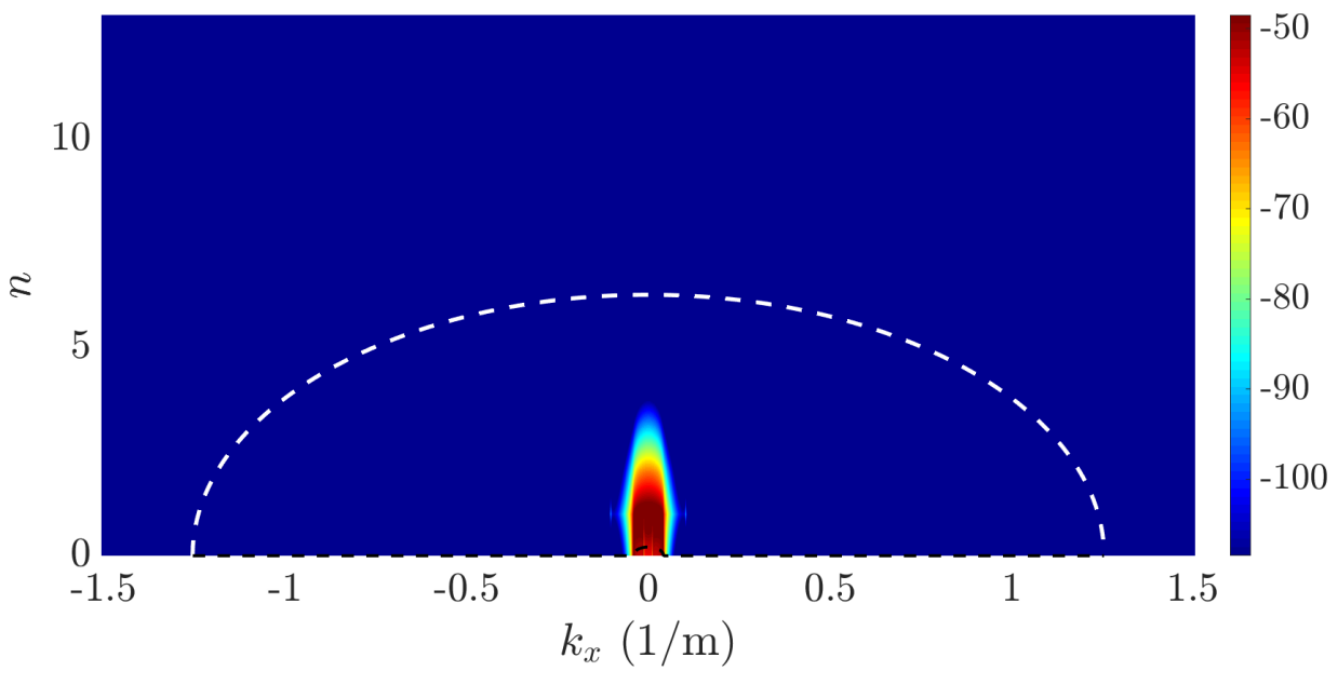

(b)

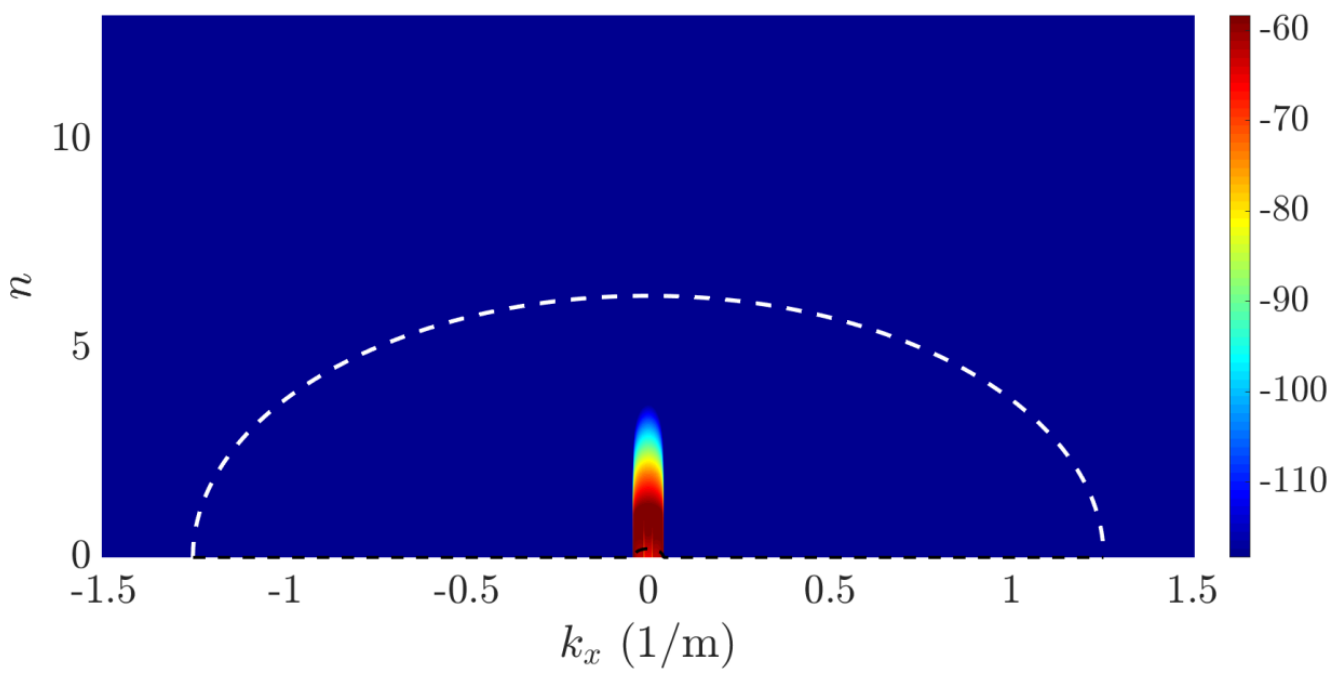

(c)

Figure 7. Circumferential sensitivity functions at a low frequency of $10 \mathrm{~Hz}$ at radial distances of (a) $\mathrm{z}=10 \mathrm{~m}$, (b) $z=100 \mathrm{~m}$ and (c) $z=1000 \mathrm{~m}$ 


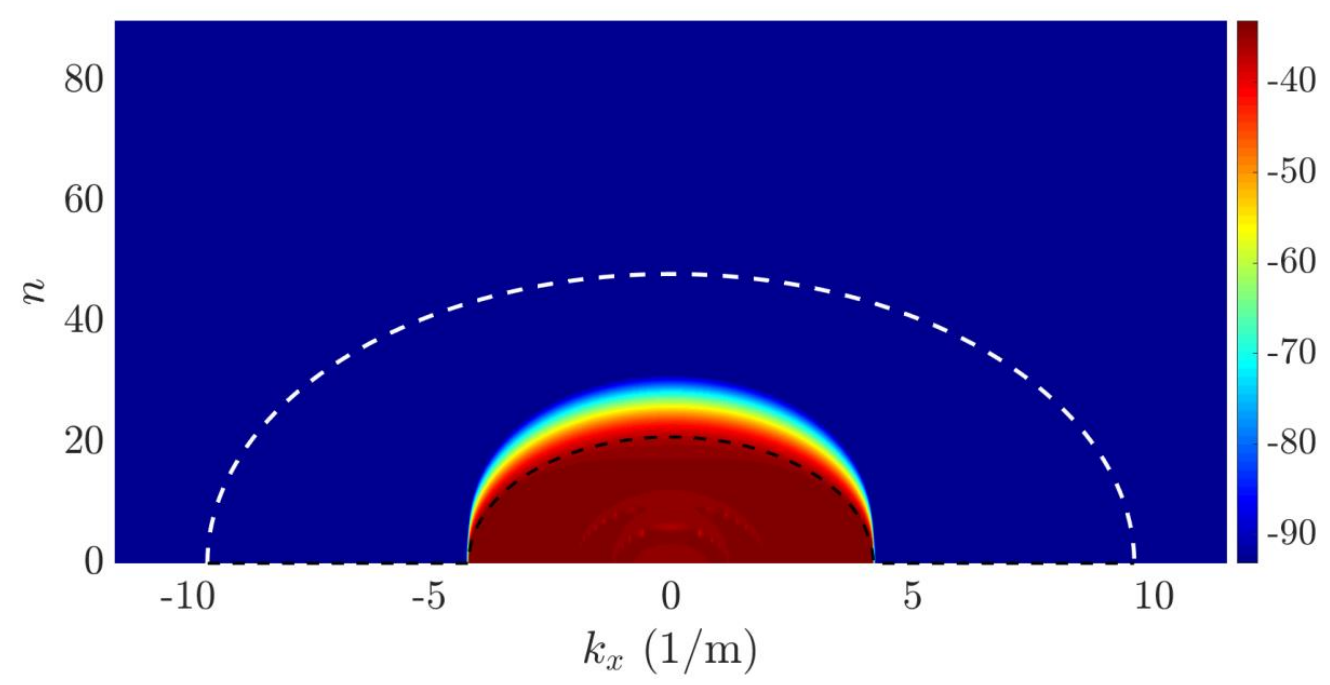

(a) $z=10 \mathrm{~m}$

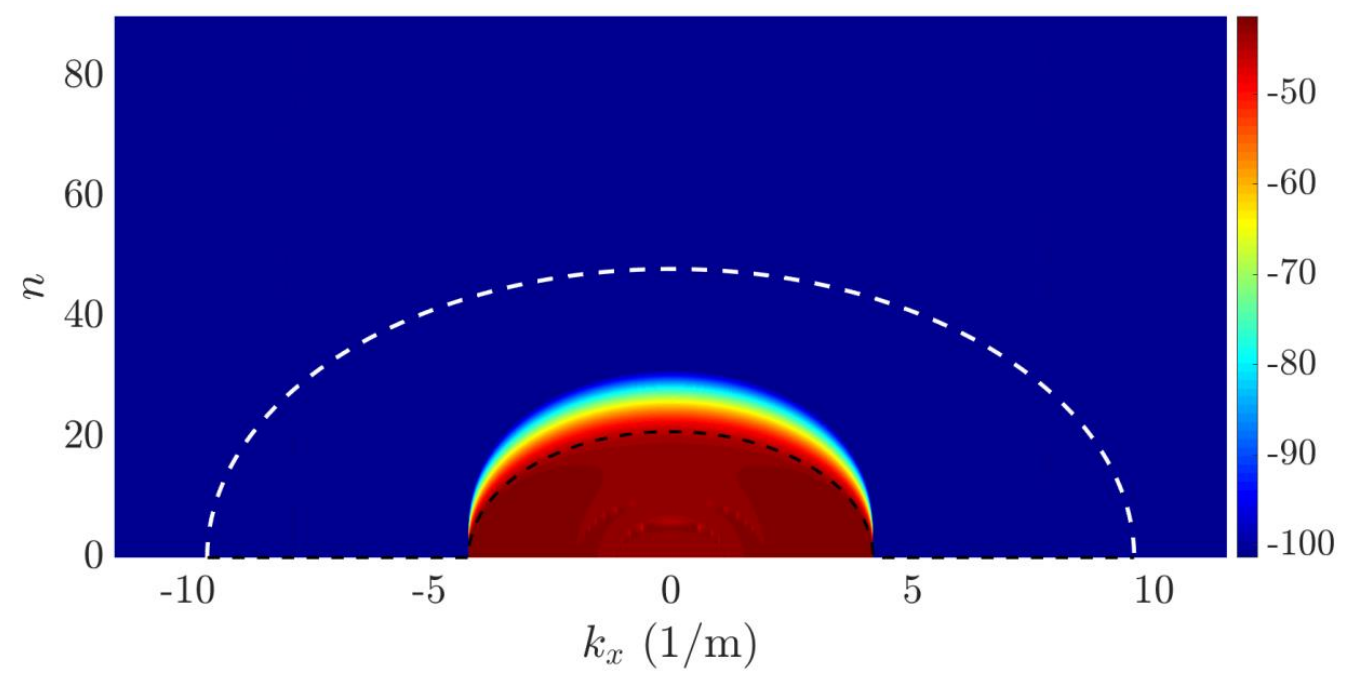

(b) $z=100 \mathrm{~m}$

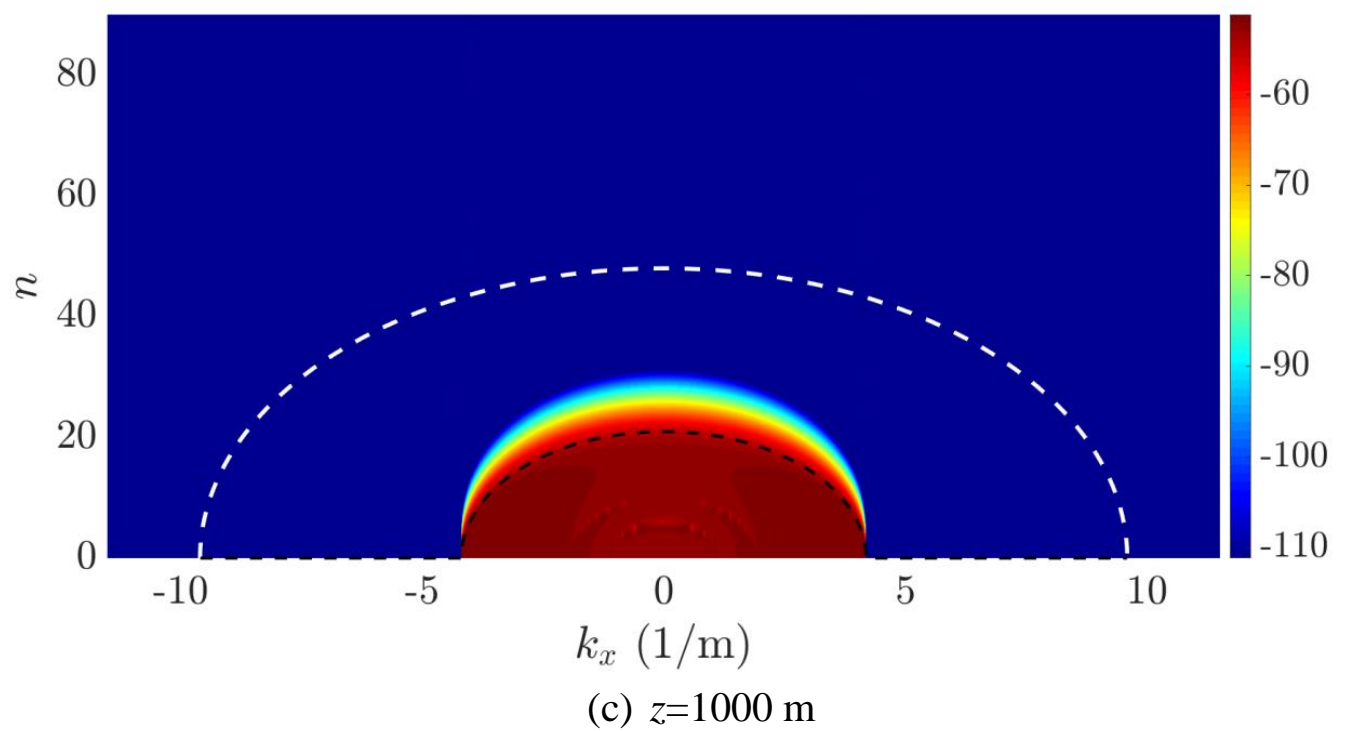

Figure 8. Circumferential sensitivity functions at a high frequency of $1000 \mathrm{~Hz}$ at radial distances of (a) $z=10 \mathrm{~m}$, (b) $z=100 \mathrm{~m}$ and (c) $z=1000 \mathrm{~m}$ 


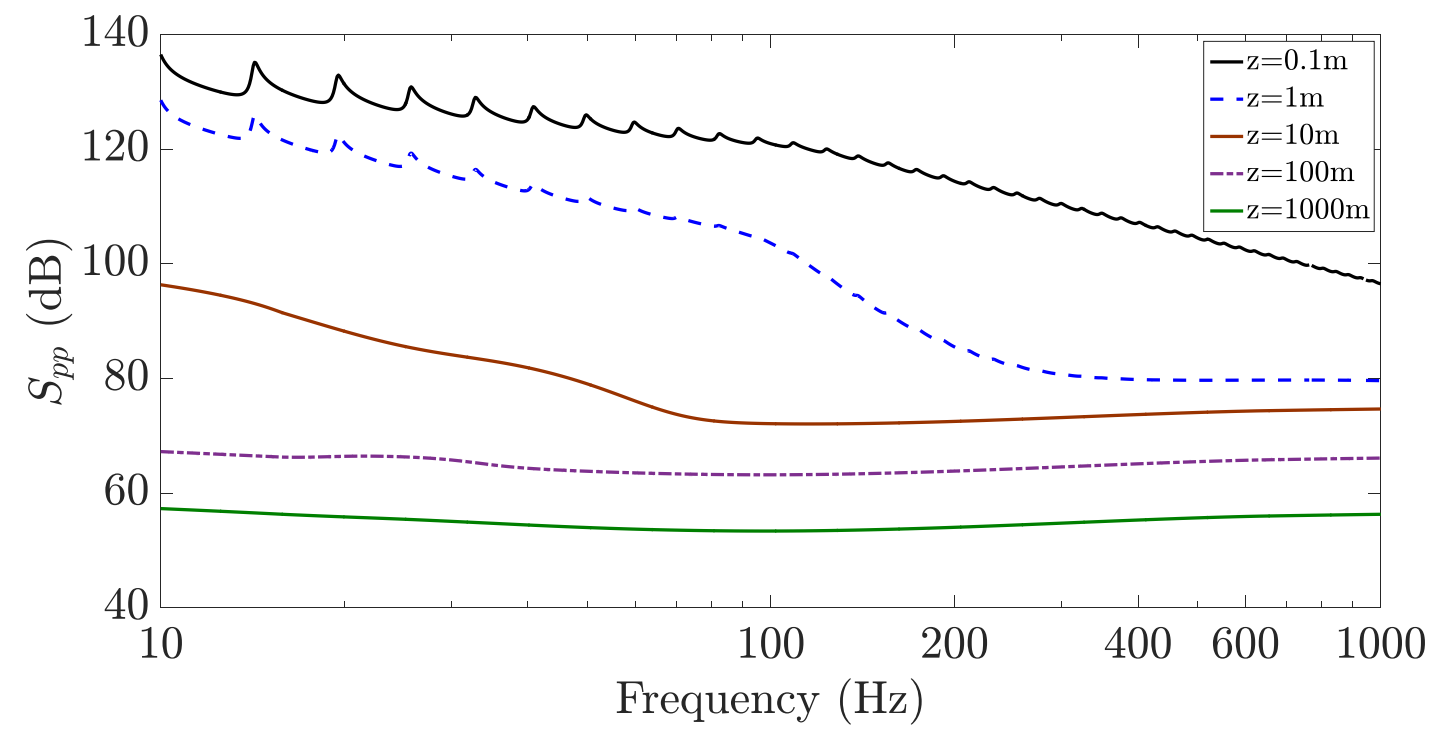

Figure 9. Auto spectral density of the acoustic pressure at different radial distances from the shell surface (dB ref. $1 \mu \mathrm{Pa} / \sqrt{\mathrm{Hz}}$ )

\subsection{Influence of the shell radius and flow speed}

Figure 10 presents the radiated pressure spectra in the far-field $(\mathrm{z}=1000 \mathrm{~m})$ for different shell radii, showing a decrease in radiated pressure with an increase in shell radius. Figure 11 presents the pressure spectra at the three shell radii considered here and the corresponding contributions by individual circumferential modes. In Figure 10, the first two peaks for $\mathrm{R}=0.5 \mathrm{~m}$ occurring at $109 \mathrm{~Hz}$ and $321 \mathrm{~Hz}$ correspond to the $\mathrm{n}=2$ and $\mathrm{n}=3$ modes and the hump occurring approximately at $600 \mathrm{~Hz}$ corresponds to the $\mathrm{n}=1$ circumferential mode, as shown in Figure 11(a). Similarly, the two visible peaks for $\mathrm{R}=1 \mathrm{~m}$ occurring at $24 \mathrm{~Hz}$ and $71 \mathrm{~Hz}$ correspond to the $\mathrm{n}=2$ and $\mathrm{n}=3$ modes, as shown in Figure 11(b). Figure 11 shows that the cut-on frequency of the shell circumferential modes for $\mathrm{n} \geq 2$ decreases with increasing shell radius. The $\mathrm{n}=0,1$ modes do not exhibit this cut-on effect (as discussed previously in Figure 4).

Figure 12 shows the effect of flow speed on the radiated sound in the far-field $(z=1000$ $\mathrm{m}$ ) for a cylindrical shell with a radius of $\mathrm{R}=5 \mathrm{~m}$. As shown in Table 1, three flow speeds were considered. The boundary layer thickness and friction velocity were assumed for 
flow speed of $6 \mathrm{~m} / \mathrm{s}$ and a corresponding equivalent distance for TBL development on a flat surface was estimated based on theoretical formula for a planar structure given by Cengel and Cimbala (2006). The equivalent distance was then used for estimation of the boundary layer thickness and friction velocity for flow speeds of $12 \mathrm{~m} / \mathrm{s}$ and $24 \mathrm{~m} / \mathrm{s}$. These TBL parameters were used in the Chase model to evaluate the CSD function of the WPF. As expected, the radiated sound increases with an increase in the flow speed. Above 20 $\mathrm{Hz}$, the radiated pressure increases by around $15 \mathrm{~dB}$ when the flow speed doubles from 6 $\mathrm{m} / \mathrm{s}$ to $12 \mathrm{~m} / \mathrm{s}$, whereas it only increases by around $7 \mathrm{~dB}$ when the flow speed doubles from $12 \mathrm{~m} / \mathrm{s}$ to $24 \mathrm{~m} / \mathrm{s}$. This is attributed to the significant increase in the friction velocity for a flow speed from $6 \mathrm{~m} / \mathrm{s}$ to $12 \mathrm{~m} / \mathrm{s}$, compared to its increase from $12 \mathrm{~m} / \mathrm{s}$ to $24 \mathrm{~m} / \mathrm{s}$ (see Table 1). The increase of the friction velocity leads to an increase of the ASD of the wall pressure field induced by the TBL, which in turn leads to an increase of the radiated pressure.

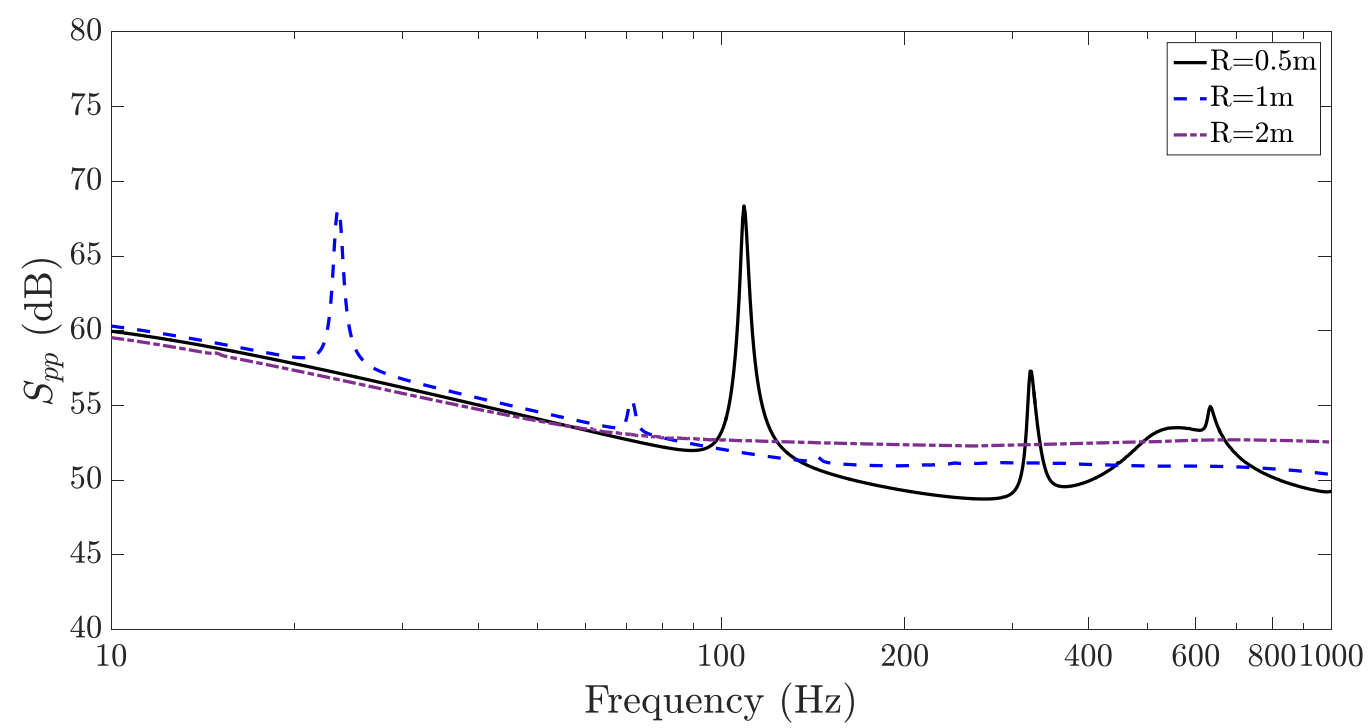

Figure 10. Auto spectral density of the acoustic pressure for different shell radii in the far field $(z=1000 \mathrm{~m})$ 


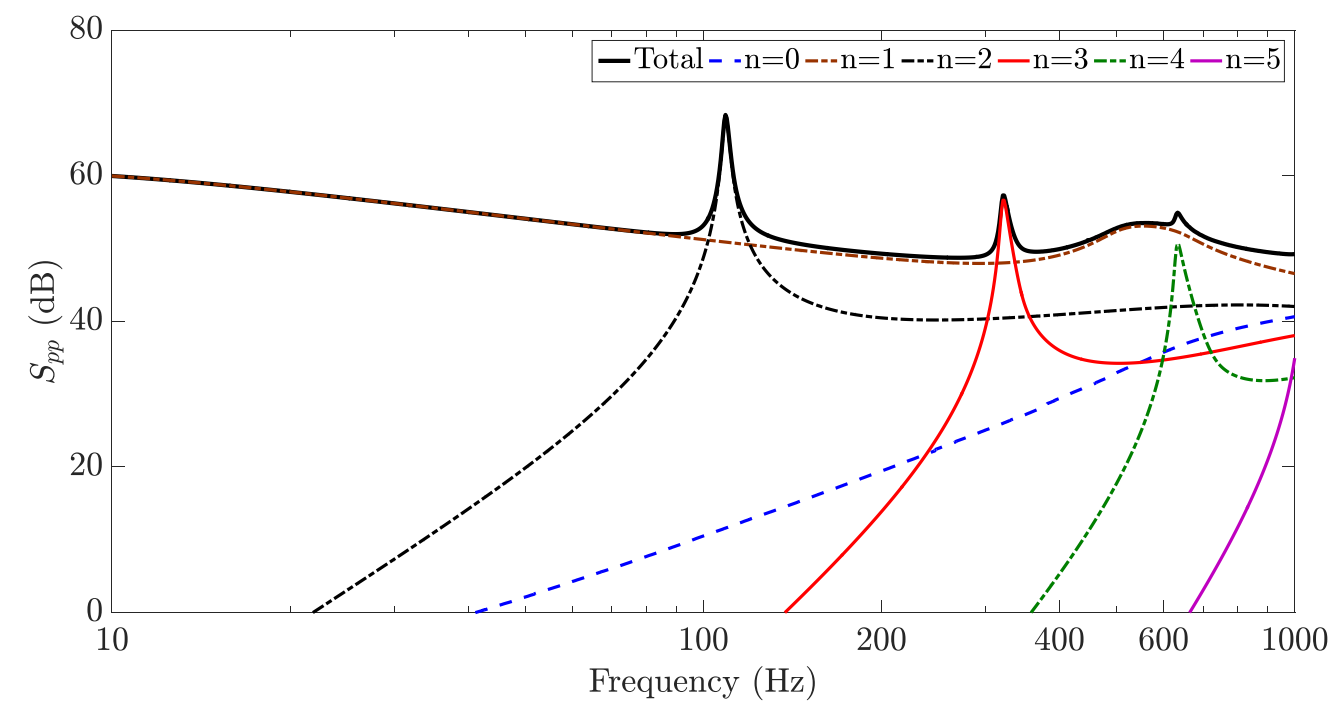

(a)

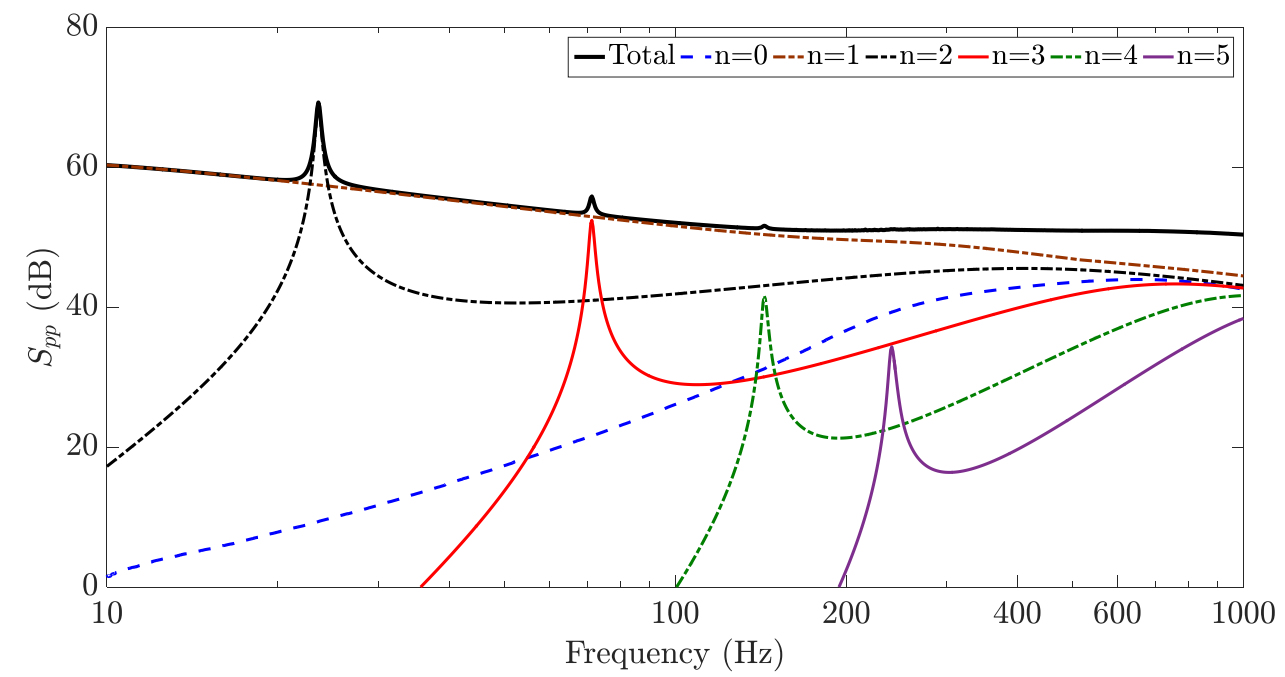

(b)

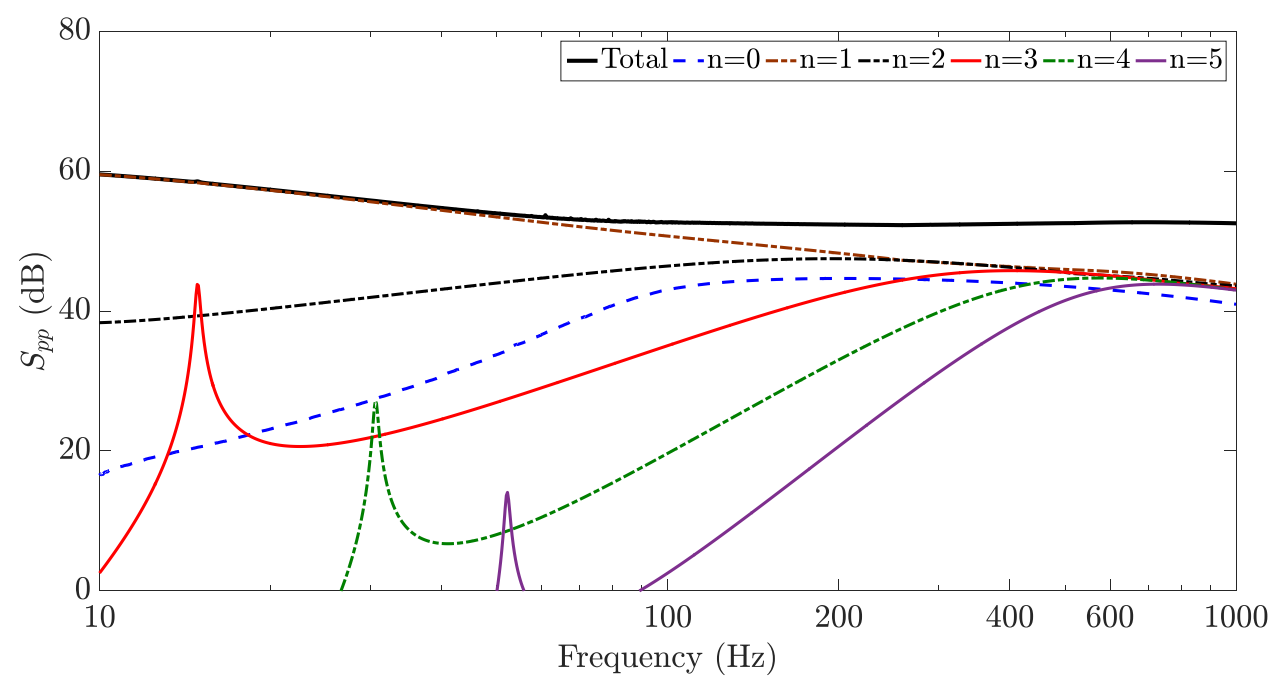

(c)

Figure 11. Auto spectral density of the acoustic pressure for different shell radii in the far field ( $z=1000 \mathrm{~m}$ ); (a) $R=0.5 \mathrm{~m}$, (b) $R=1 \mathrm{~m}$, (c) $R=2 \mathrm{~m},(\mathrm{~dB}$ ref. $1 \mu \mathrm{Pa} / \sqrt{\mathrm{Hz}}$ ). 
Table 1. Boundary layer parameters used to compute the ASD in Figure 12

\begin{tabular}{ccc}
\hline $\begin{array}{c}\text { Free stream velocity } \\
U_{\infty}(\mathrm{m} / \mathrm{s})\end{array}$ & $\begin{array}{c}\text { Boundary layer thickness } \\
\delta(\mathrm{m})\end{array}$ & \begin{tabular}{c}
$v_{\tau}(\mathrm{m} / \mathrm{s})$ \\
\hline 6
\end{tabular} \\
12 & 0.15 & 1 \\
24 & 0.13 & 1.87 \\
\hline
\end{tabular}

Figure 13 presents the simulation run time to compute the radiated sound using the present approach as a function of frequency for the cylinder with a radius of $\mathrm{R}=5 \mathrm{~m}$. Simulations were conducted using MATLAB on a desktop personal computer with $32 \mathrm{~GB}$ of random access memory (RAM) and a total of four physical cores running at $3.2 \mathrm{GHz}$. It can be seen from Figure 13 that as the frequency increases, the computational time increases dramatically. This is attributed to the fact that more wavenumbers and circumferential modes need to be considered in the computation at high frequencies. A linear function corresponding to $0.0039 f-0.0324$ was curve fitted to the simulation run time data and is also shown in Figure 13, clearly highlighting that the run time varies linearly with frequency. For the cylindrical shell model considered here, the maximum run time for the highest frequency of interest at $1 \mathrm{kHz}$ is less than 4 seconds. 


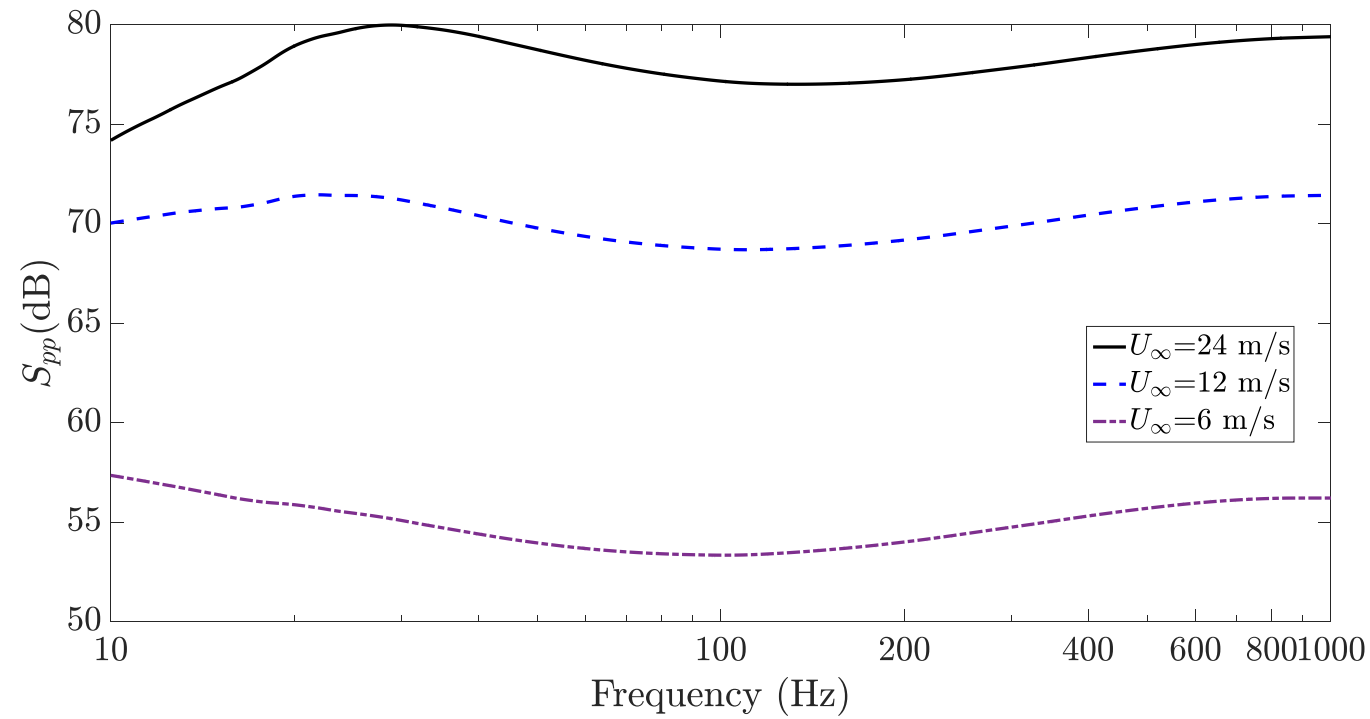

Figure 12. Auto spectral density of the acoustic pressure in the far field $(z=1000 \mathrm{~m})$ for different flow speeds.

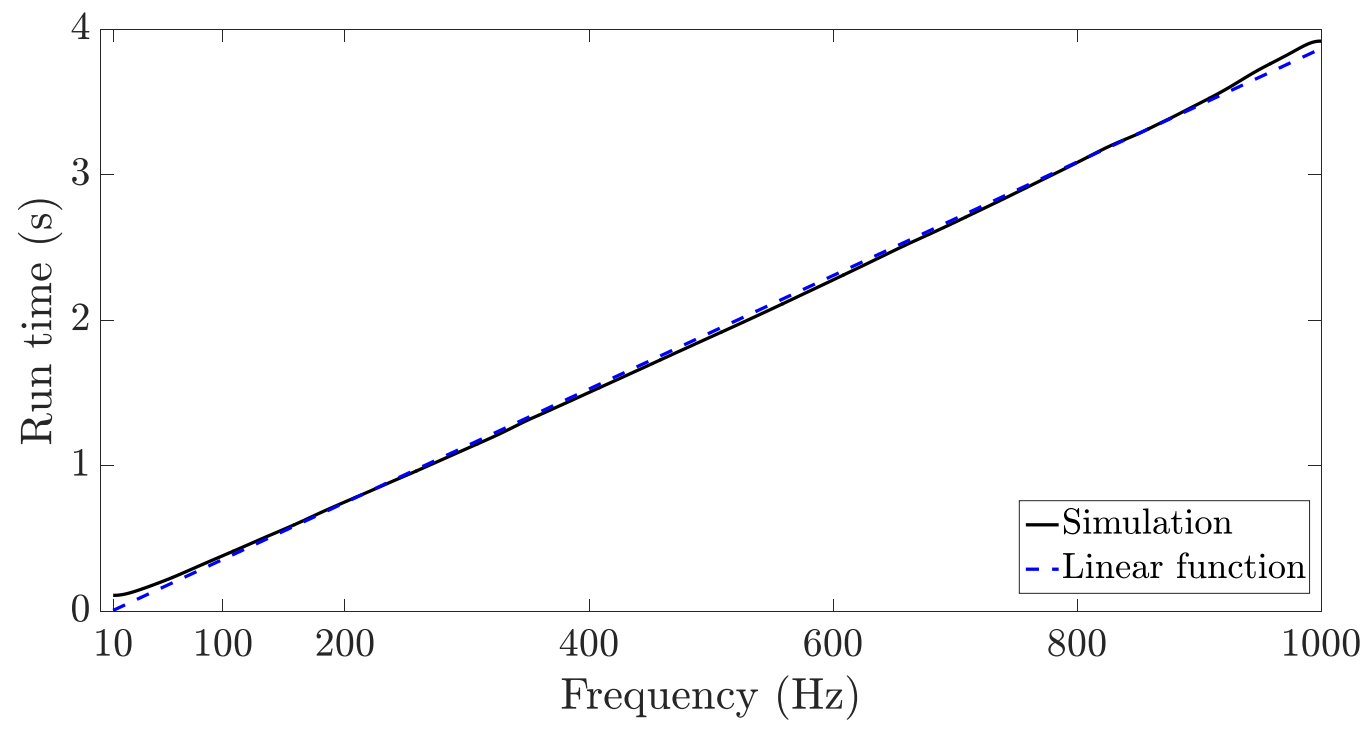

Figure 13. Computational run time to compute the radiated acoustic pressure 


\section{Summary}

An analytical model of a fluid loaded cylindrical shell excited by a turbulent flow field has been presented. A wavenumber-point reciprocity principle was implemented to identify circumferential sensitivity functions. The Chase model was used to describe the wall pressure field induced by the turbulent boundary layer in the wavenumber-frequency domain. Our proposed method provides the ability to investigate the physical mechanisms associated with the noise radiated by a cylindrical shell excited by a TBL, by examining the contributions by individual circumferential modes to the acoustic spectra. The pressure spectra in both in the near field and far field were presented for frequencies below and above the ring frequency, as well as for different shell radii and flow speeds. The proposed method is also an efficient computational tool for evaluation of the vibroacoustic responses of a cylindrical shell under flow excitation, typically found in maritime applications. In the near future, we will implement our numerical approach to study more realistic designs of a submerged marine vessel that incorporate the effects of ring stiffeners and other internal structures of the hull.

\section{Acknowledgement}

This work was carried out in the framework of the LabEx CeLyA ("Centre Lyonnais d'Acoustique", ANR-10-LABX-60). The second author gratefully acknowledges financial support from the Australian Government through the Australian Research Council's Discovery Projects funding scheme (project DE190101412). 


\section{Appendix A}

The elements of the spectral Flügge operator $\mathcal{L}(x, \theta)$ are given by (Karczub, 2006)

$$
\begin{gathered}
\mathcal{L}(x, \theta)=\left\{\begin{array}{lll}
Z_{U U} & Z_{U V} & Z_{U W} \\
Z_{U V} & Z_{V V} & Z_{V W} \\
Z_{U W} & Z_{V W} & Z_{W W}
\end{array}\right\}, \\
Z_{U U}=R^{2} \frac{\partial^{2}}{\partial x^{2}}-\rho_{s} R^{2} \frac{1-v^{2}}{E} \frac{\partial^{2}}{\partial t^{2}}+\left(1+\beta^{2}\right) \frac{1-v}{2} \frac{\partial^{2}}{\partial \theta^{2}}, \\
Z_{U V}=R \frac{1+v}{2} \frac{\partial^{2}}{\partial x \partial \theta}, \\
Z_{U W}=R v \frac{\partial}{\partial x}-\beta^{2} R^{3} \frac{\partial^{3}}{\partial x^{3}}+\beta^{2} R \frac{1-v}{2} \frac{\partial^{3}}{\partial x \partial \theta^{2}}, \\
Z_{V W}=\left(1+3 \beta^{2}\right) R^{2} \frac{1-v}{2} \frac{\partial^{2}}{\partial x^{2}}+\frac{\partial^{2}}{\partial \theta^{2}}-\rho_{s} R^{2} \frac{1-v^{2}}{E} \frac{\partial^{2}}{\partial t^{2}}, \\
Z_{V W}=\frac{\partial}{\partial \theta}-\beta^{2} R^{2} \frac{3-v}{2} \frac{\partial^{3}}{\partial x^{2} \partial \theta}, \\
Z_{W W}=1+\beta^{2}\left(R^{4} \frac{\partial^{4}}{\partial x^{4}}+2 R^{2} \frac{\partial^{4}}{\partial x^{2} \partial \theta^{2}}+\frac{\partial^{4}}{\partial \theta^{4}}\right)+\rho_{s} R^{2} \frac{1-v^{2}}{E} \frac{\partial^{2}}{\partial t^{2}}+\beta^{2}\left(1+2 \frac{\partial^{2}}{\partial \theta^{2}}\right),
\end{gathered}
$$

where $\beta=\frac{h}{\sqrt{12} R}$ is the shell thickness parameter. 


\section{Appendix B}

The elements of the spectral Flügge operator $\tilde{\mathcal{L}}\left(k_{x}, n\right)$ in the wavenumber domain are given by

$$
\begin{aligned}
& \tilde{\mathcal{L}}\left(k_{x}, n\right)=\left[\begin{array}{lll}
\tilde{Z}_{U U} & \tilde{Z}_{U V} & \tilde{Z}_{U W} \\
\tilde{Z}_{U V} & \tilde{Z}_{V V} & \tilde{Z}_{V W} \\
\tilde{Z}_{U W} & \tilde{Z}_{V W} & \tilde{Z}_{W W}
\end{array}\right], \\
& \tilde{Z}_{U U}=R^{2}\left(k_{l}^{2}-k_{x}^{2}\right)-n^{2} \frac{1-v}{2}\left(1+\beta^{2}\right), \\
& \tilde{Z}_{U V}=-n R \frac{1+v}{2} k_{x}, \\
& \tilde{Z}_{U W}=\mathrm{i} k_{x}\left(R v-\beta^{2}\left(-R^{3} k_{x}^{2}+n^{2} R \frac{1-v}{2}\right)\right), \\
& \tilde{Z}_{V V}=-R^{2} \frac{1-v}{2} k_{x}^{2}\left(1+3 \beta^{2}\right)-n^{2}+R^{2} k_{l}^{2}, \\
& \tilde{Z}_{V W}=\mathrm{i} n\left(1+\beta^{2} R^{2} \frac{3-v}{2} k_{x}^{2}\right), \\
& \tilde{Z}_{W W}=1+\beta^{2}\left(R^{4} k_{x}^{4}+2 n^{2}\left(R^{2} k_{x}^{2}+1\right)+n^{4}+1\right)-R^{2} k_{l}^{2},
\end{aligned}
$$

where $k_{l}=\omega \sqrt{\frac{\rho_{s}\left(1-v^{2}\right)}{E}}$ is the wavenumber for longitudinal waves propagating in the cylindrical shell. 


\section{Appendix C}

The cross spectral density of the Chase TBL model for a planar structure is given by (Chase, 1987)

$\varphi_{p p}\left(k_{x}, k_{y}, \omega\right)=\frac{(2 \pi)^{3} \rho_{f}{ }^{2} v_{\tau}{ }^{3}}{\left(K_{+}^{2}+(b \delta)^{-2}\right)^{5 / 2}}\left(C_{M} k_{x}{ }^{2}+C_{T} K^{2}\left(\frac{K_{+}^{2}+(b \delta)^{-2}}{K^{2}+(b \delta)^{-2}}\right)\right)$

with $K_{+}^{2}=\left(\omega-U_{c} k_{x}\right)^{2} /\left(q v_{\tau}\right)^{2}+K^{2}$ and $K^{2}=k_{x}^{2}+k_{y}^{2}$. Recommended parameters for $b, q$, $C_{M}, C_{T}$ are given as $b=0.75, q=3, C_{M} \approx 0.1553$ and $C_{T} \approx 0.0047$ (Howe, 1998).

\section{REFERENCES}

Chase, D.M., 1987. The character of the turbulent wall pressure spectrum at subconvective wavenumbers and a suggested comprehensive model. J. Sound Vib. 112(1), 125-147.

Cengel, Y., Cimbala, J., 2006. Fluid Mechanics: Fundamentals and Applications. McGraw-Hill series in mechanical engineering, Mc Graw-Hill Higher Education.

Ciappi, E., De Rosa, S., Franco, F., Guyader, J.L., Hambric, S.A., 2014. Flinovia-flow induced noise and vibration issues and aspects, Springer International Publishing Switzerland.

Ciappi, E., De Rosa, S., Franco, F., Guyader, J.L., Hambric, S.A., Leung, R.C.K. and Hanford, A.D., 2018. Flinovia-Flow Induced Noise and Vibration Issues and Aspects-II. Springer International Publishing Switzerland.

Ciappi, E., Magionesi, F., De Rosa, S. and Franco, F., 2009. Hydrodynamic and hydroelastic analyses of a plate excited by the turbulent boundary layer. J. Fluids Struc, 25(2), 321-342.

Ciappi, E., De Rosa, S., Franco, F., Vitiello, P. and Miozzi, M., 2016. On the dynamic behavior of composite panels under turbulent boundary layer excitations. J. Sound Vib. 364, 77-109.

Corcos, G. M., 1963. Resolution of pressure in Turbulence, J. Acoust. Soc. Am. 35, 192-199.

Crocker, M.J., 1998. Handbook of acoustics. John Wiley \& Sons. 
Davis, H. G., 1971. Sound from turbulent boundary layer excited panel, J. Acoust. Soc. Am. 49, 878-889.

De Rosa, S. and Franco, F., 2008. Exact and numerical responses of a plate under a turbulent boundary layer excitation. J. Fluids Struc. 24(2), 212-230.

Durant, C., Robert, G., Filippi, P.J.T., Mattei, P.O., 2000. Vibroacoustic response of a thin cylindrical shell excited by a turbulent internal flow: comparison between numerical prediction and experimentation. J. Sound Vib. 229(5), 1115-1155.

Errico, F., Ichchou, M., Franco, F., De Rosa, S., Bareille, O. and Droz, C., 2019. Schemes for the sound transmission of flat, curved and axisymmetric structures excited by aerodynamic and acoustic sources. J. Sound Vib. 456 (15), 221-238.

Fahy, F.J. and Gardonio, P., 2006. Sound and structural vibration: Radiation, transmission and response. Academic Press.

Goody, M. C., 2004. Empirical spectral model of surface pressure fluctuations, AIAA J., 42, 1788-1794.

Hambric, S.A., Hwang, Y.F. and Bonness, W.K., 2004. Vibrations of plates with clamped and free edges excited by low-speed turbulent boundary layer flow. J. Fluid Struct. 19(1), 93-110.

Howe, M.S., 1998. Acoustics of fluid-structure interactions. Cambridge University Press.

James, J.H., 1982. Computation of Acoustic Power, Vibration Response and Acoustic pressures of fluid-filled pipes. Admiralty marine technology establishment Teddington (England). no. 82036.

Junger, M.C. and Feit, D., 1986. Sound, structures, and their interaction, Cambridge, MA: MIT press.

Karczub, D.G., 2006. Expressions for direct evaluation of wave number in cylindrical shell vibration studies using the Flügge equations of motion. J. Acoust. Soc. Am. 119(6), 3553-3557.

Leissa, A.W., 1973. Vibration of shells. Washington: Scientific and Technical Information Office, National Aeronautics and Space Administration. 
Li, Y., Zhang, Y. and Kennedy, D., 2017. Random vibration analysis of axially compressed cylindrical shells under turbulent boundary layer in a symplectic system. J. Sound Vib. 406, 161-180.

Liu, B., 2008. Noise radiation of aircraft panels subjected to boundary layer pressure fluctuations. J. Sound Vib. 314, 693-711.

Lueptow, R.M., 1988. Turbulent boundary layer on a cylinder in axial flow, Technical report, DTIC Document.

Maxit, L. and Ginoux, J.M., 2010. Prediction of the vibro-acoustic behavior of a submerged shell non periodically stiffened by internal frames. J. Acoust. Soc. Am. 128(1), 137-151.

Marchetto, C., Maxit, L., Robin, O. and Berry, A., 2017. Vibroacoustic response of panels under diffuse acoustic field excitation from sensitivity functions and reciprocity principles. J. Acoust. Soc. Am. 141(6), 4508-4521.

Marchetto, C., Maxit, L., Robin, O. and Berry, A., 2018. Experimental prediction of the vibration response of panels under a turbulent boundary layer excitation from sensitivity functions. J. Acoust. Soc. Am. 143(5), 2954-2964.

Maxit, L. and Denis, V., 2013. Prediction of flow induced sound and vibration of periodically stiffened plates. J. Acoust. Soc. Am. 133(1), 146-160.

Maxit, L., 2016. Simulation of the pressure field beneath a turbulent boundary layer using realizations of uncorrelated wall plane waves, J. Acoust. Soc. Am. 140, 1268-1285.

Maury, C., Gardonio, P. and Elliott, S.J., 2002a. A wavenumber approach to modelling the response of a randomly excited panel, part I: general theory. J. Sound Vib. 252(1), 83-113.

Maury, C., Gardonio, P. and Elliott, S.J., 2002b. A wavenumber approach to modelling the response of a randomly excited panel, Part II: Application to aircraft panels excited by a turbulent boundary layer. J. Sound Vib. 252(1), 115-139.

Mazzoni, D., 2003. An efficient approximation for the vibroacoustic response of a turbulent boundary layer excited panel, J. Sound Vib. 264, 951-971.

Norton, M.P. and Bull, M.K., 1984. Mechanisms of the generation of external acoustic radiation from pipes due to internal flow disturbances. J. Sound Vib. 94(1), 105-146. 
Photiadis, D.M., 1990. The propagation of axisymmetric waves on a fluid-loaded cylindrical shell, J. Acoust. Soc. Am. 88, 239-250.

Strawderman, W.A., 1969. Turbulence-induced plate vibrations: An evaluation of finite- and infinite-plate models, J. Acoust. Soc. Am. 46, 1294-1295.

Strawderman, W.A., Christman, R.A., 1971. Turbulence-induced plate vibrations: Some effects of fluid loading on finite and infinite plates, J. Acoust. Soc. Am. 52, 1537-1552.

Scott, J.F.M., 1988. The free modes of propagation of an infinite fluid-loaded thin cylindrical shell. J. Sound Vib. 125(2), 241-280.

Tang, Y., Silcox, R., Robinson, J., 1996. Sound transmission through cylindrical shell structures excited by boundary layer pressure fluctuations. In Aeroacoust. Conf. p. 1760.

Williams, E.G., Houston, B.H., Bucaro, J.A., 1990. Experimental investigation of the wave propagation on a point-driven, submerged capped cylinder using $K$-space analysis, J. Acoust. Soc. Am. 87, 513-552.

Williams, E.G., 1999. Fourier acoustics: sound radiation and nearfield acoustical holography. Academic press, Elsevier.

Zhang, Q., Mao, Y. and Qi, D., 2018. Analytical modeling of the vibro-acoustic response of a double-walled cylindrical shell with microperforation excited by turbulent boundary layer pressure fluctuations. J. Vib. Acoust, 140(2), 1-13.

Zhou, J., Bhaskar, A. and Zhang, X., 2015. Sound transmission through double cylindrical shells lined with porous material under turbulent boundary layer excitation. J. Sound Vib. 357, 253-268. 